\title{
Effect of external magnetic fields on nucleon mass in a hot and dense medium: Inverse magnetic catalysis in the Walecka model
}

\author{
Arghya Mukherjee, ${ }^{1,2, *}$ Snigdha Ghosh, ${ }^{3,2,4, \dagger}$ Mahatsab Mandal, ${ }^{5, \$}$ Sourav Sarkar, ${ }^{3,2, \S}$ and Pradip Roy ${ }^{1,2, \|}$ \\ ${ }^{1}$ Saha Institute of Nuclear Physics, 1/AF Bidhannagar, Kolkata 700064, India \\ ${ }^{2}$ Homi Bhabha National Institute, Training School Complex, Anushaktinagar, Mumbai 400085, India \\ ${ }^{3}$ Variable Energy Cyclotron Centre, I/AF Bidhannagar, Kolkata 700 064, India \\ ${ }^{4}$ Indian Institute of Technology Gandhinagar, Palaj, Gandhinagar 382355, Gujarat, India \\ ${ }^{5}$ Government General Degree College at Kalna-I, Burdwan, West Bengal 713405, India
}

(Received 26 July 2018; published 26 September 2018)

\begin{abstract}
The vacuum to nuclear matter phase transition is studied in the presence of a constant external background magnetic field with the mean-field approximation in the Walecka model. The anomalous nucleon magnetic moment is taken into account using the modified "weak" field expansion of the fermion propagator with nontrivial correction terms for both charged and neutral particles. The effect of the nucleon magnetic moment is found to favor the magnetic catalysis effect at zero temperature and zero baryon density. However, extending the study to finite temperatures, it is observed that the anomalous nuclear magnetic moment plays a crucial role in characterizing the qualitative behavior of the vacuum to nuclear matter phase transition even in the case of weak external magnetic fields. The critical temperature corresponding to the vacuum to nuclear medium phase transition is observed to decrease with the external magnetic field, which can be identified as inverse magnetic catalysis in the Walecka model, whereas the opposite behavior is obtained in the case of a vanishing magnetic moment, indicating magnetic catalysis.
\end{abstract}

DOI: 10.1103/PhysRevD.98.056024

\section{INTRODUCTION}

Understanding QCD in the presence of a magnetic background has recently gained a lot of attention [1]. It is important to study QCD in the presence of an external magnetic field not only for its relevance to astrophysical phenomena [2-8], but also due to the possibility of strong magnetic field generation in noncentral heavy-ion collisions [9], which sets the stage for the investigation of these magnetic modifications. Although the background fields produced at RHIC and LHC are much smaller compared to the field strengths present during the cosmological electroweak phase transition (which may reach up to $e B \approx 200 m_{\pi}^{2}$ [10]), they are strong enough to have a significant influence on hadronic properties that carry information about the chiral phase transition. At vanishing chemical potential, modifications due to the presence of a magnetic

\footnotetext{
*arghya.mukherjee@saha.ac.in

†nigdha.physics@gmail.com, snigdha.ghosh@iitgn.ac.in

*mahatsab@gmail.com

\$sourav@vecc.gov.in

pradipk.roy@saha.ac.in
}

Published by the American Physical Society under the terms of the Creative Commons Attribution 4.0 International license. Further distribution of this work must maintain attribution to the author(s) and the published article's title, journal citation, and DOI. Funded by SCOAP. background can be obtained from first principles using lattice QCD simulations [11,12], which show a monotonic increase in the critical temperature with increasing magnetic field. In recent years, the effects of an external magnetic field on the chiral phase transition have been studied using different effective models [13-25]. As QCD is a confining theory at low energies, effective theories are employed to describe the low-energy behavior of the strong interaction. In such a theory, the condensate is described as the nonzero expectation value of the sigma field, which is basically a composite operator of two quark fields. If the condensate is already present without any background field, the effect of its enhancement in the presence of an external magnetic field is called magnetic catalysis (MC). Effective field-theoretic models in general contain a few parameters that can be fixed from experimental inputs. Although most of the model calculations support MC, some lattice results have shown inverse magnetic catalysis (IMC), where the critical temperature follows the opposite trend [26-29]. It was pointed out in Ref. [30] that IMC is attributed to the dominance of the sea contribution over the valence contribution of the quark condensate. The sea effect has not been considered even in the Polyakov-loopextended versions of the Nambu-Jona-Lasinio (NJL) and quark-meson models, which might be a possible reason for the disagreement. To investigate the apparent contradiction, a significant amount of work has been done [31] to find 
proper modifications of the effective models, most of which is focused on the magnetic field dependence of the coupling constants or other magnetic-field-dependent parameters in the model. Very recently, IMC was observed in the NJL model with the Pauli-Villars regularization scheme [32], which gives markedly different behavior in comparison to the usual soft-cutoff approach.

In the context of nuclear physics, the MC effect was discussed by Haber et al. in Ref. [33]. There, the effect of a background magnetic field on the transition between vacuum and nuclear matter at zero temperature was studied for the Walecka model [34] as well as for the extended linear sigma model. The study included the $B$-dependent Dirac sea contribution of the free energy density which was previously ignored in the case of magnetized nuclear matter (see, e.g., Refs. [35-43]). Following a renormalization procedure similar to the case of magnetized quark matter, the cutoff dependence of the $B$-dependent sea contribution is absorbed into a renormalized magnetic field and a renormalized electric charge. The onset of the vacuum to nuclear matter phase transition is determined by equating the corresponding free energies. From the qualitative agreement between the two models, it is evident that with the proper incorporation of the magnetic catalysis effect the creation of nuclear matter becomes energetically more expensive in the presence of a background magnetic field. However, there is an important qualitative difference between the two models. As the analysis suggests, in the Walecka model there is a region where the critical chemical potential for the vacuum to nuclear matter transition is lower than that in the absence of a background field. This feature has surprising similarities with the IMC present in the NJL and holographic Sakai-Sugimoto models [44]. It would be interesting to see whether a similar feature also exists in a more generalized scenario. Now, as the anomalous magnetic moment (AMM) of the nucleons has not been taken into account in the analysis, an obvious generalization will be to incorporate it into the study of the vacuum to nuclear matter phase transition under an external magnetic field at nonzero temperature. However, a recent study [45] incorporating the magnetic-fielddependent vacuum at finite temperature and density showed that the AMM of charged fermions makes no significant contribution to the equation of state at any external field value. Thus, it will be interesting to see whether MC persists in the presence of an anomalous magnetic moment.

In this work we restrict ourselves to the "weak" field regime of the external magnetic field and use the Walecka model to describe the nucleon-nucleon interaction. In this model, the interaction between the nucleons is described by the exchange of scalar $(\sigma)$ and vector $(\omega)$ mesons. A more realistic extension of the Walecka model where the selfinteractions of the meson fields are also considered is ignored here for the sake of simplicity, as they hardly contribute to the qualitative nature of the results presented in this work. Now, to obtain the effective mass of the nucleons, instead of minimizing the free energy density with respect to the condensate [33], we calculate the effective nucleon propagator by summing up the scalar and vector tadpole diagrams self-consistently. In that case, the effective mass of the nucleon appears as a pole of the effective nucleon propagator. In the case of a weak magnetic field, the nucleon propagators can be expressed as a series in powers of $q B$ and $\kappa B$, where $q$ and $\kappa$ represent the charge and anomalous magnetic moment of the nucleons. It should be mentioned here that we employ the mean-field approximation in the calculation of the tadpole diagrams using the interacting propagator. It is essentially equivalent to solving the meson field equations with the replacement of the meson field operators by their expectation values. In other words, under this approximation, the meson field operators become classical fields that are assumed to be uniform in space and time, and the fluctuation around this background is neglected.

The article is organized as follows. In Sec. II, the familiar expression [46] for the weak-field expansion of the charged scalar propagator in the presence of a constant external magnetic field is derived using the perturbative method. The same procedure is employed in Sec. III to obtain the weak-field expanded propagators of the charged and neutral fermions with nonzero magnetic moment. Suitable forms of the corresponding thermal propagators are also discussed, which are used to obtain the effective mass of the nucleons in the Walecka model described in Sec. IV. Section V contains our numerical results and discussions. Finally, we summarize our work in Sec. VI.

\section{CHARGED SCALAR PROPAGATOR IN AN EXTERNAL MAGNETIC FIELD}

Let us first consider the propagation of a charged scalar particle with zero external magnetic field. In this case, the scalar vacuum Feynman propagator $\Delta_{F}\left(x, x^{\prime}\right)=\Delta_{F}\left(x-x^{\prime}\right)$ satisfies

$$
\left(\partial_{\mu} \partial^{\mu}+m^{2}\right) \Delta_{F}\left(x-x^{\prime}\right)=\delta^{4}\left(x-x^{\prime}\right) .
$$

In order to solve Eq. (1), we introduce the Fourier transform of $\Delta_{F}\left(x-x^{\prime}\right)$,

$$
\Delta_{F}\left(x-x^{\prime}\right)=\int \frac{d^{4} k}{(2 \pi)^{4}} e^{-i k \cdot\left(x-x^{\prime}\right)} \Delta_{F}(k),
$$

where $\Delta_{F}(k)$ is the momentum-space vacuum scalar propagator. Substituting $\Delta_{F}\left(x-x^{\prime}\right)$ from Eq. (2) into Eq. (1), we get

$$
\Delta_{F}(k)=\left(\frac{-1}{k^{2}-m^{2}+i \epsilon}\right),
$$

where we have imposed Feynman's boundary condition and put $i \epsilon$ in the denominator. 
We now turn on the external magnetic field. In this case, the charged scalar propagator in an external magnetic field, denoted by $\Delta_{B}\left(x, x^{\prime}\right)$, will satisfy

$$
\left[\left\{\partial_{\mu}+i q A_{\mu}(x)\right\}\left\{\partial^{\mu}+i q A^{\mu}(x)\right\}+m^{2}\right] \Delta_{B}\left(x, x^{\prime}\right)=\delta^{4}\left(x-x^{\prime}\right),
$$

where $q$ is the electric charge of the particle and $A^{\mu}(x)$ is the four-potential corresponding to the external magnetic field. We note that the propagator $\Delta_{B}\left(x, x^{\prime}\right)$ is not translation invariant. To solve Eq. (4), we follow the procedure given in Refs. $[47,48]$ and choose a particular gauge in which the four-potential is

$$
A^{\mu}(x)=-\frac{1}{2} F^{\mu \nu} x_{\nu}
$$

For the case of a constant external magnetic field, the fieldstrength tensor $F^{\mu \nu}$ is independent of $x$. Substituting Eq. (5) into Eq. (4), we get

$$
\begin{aligned}
& {\left[\square+m^{2}-i q F^{\mu \nu} x_{\nu} \partial_{\mu}-\frac{q^{2}}{4} F^{\mu \alpha} F_{\mu \beta} x_{\alpha} x^{\beta}\right] \Delta_{B}\left(x, x^{\prime}\right)} \\
& =\delta^{4}\left(x-x^{\prime}\right) \text {. }
\end{aligned}
$$

The corresponding momentum-space propagator $\Delta_{B}(k)$ is obtained from the Fourier transform of the translation-invariant part of the coordinate-space propagator $\Delta_{B}\left(x, x^{\prime}\right)$, i.e.,

$$
\Delta_{B}\left(x, x^{\prime}\right)=\phi\left(x, x^{\prime}\right) \int \frac{d^{4} k}{(2 \pi)^{4}} e^{-i k \cdot\left(x-x^{\prime}\right)} \Delta_{B}(k),
$$

where $\phi\left(x, x^{\prime}\right)$ is the phase factor, which depends on the choice of gauge. For the gauge given in Eq. (5), the phase factor is [48]

$$
\phi\left(x, x^{\prime}\right)=\exp \left[\frac{i}{2} q F^{\alpha \beta} x_{\alpha} x^{\prime \beta}\right] .
$$

Substituting Eq. (7) into Eq. (6), we get

$$
\begin{aligned}
& \int \frac{d^{4} k}{(2 \pi)^{4}} e^{-i k \cdot\left(x-x^{\prime}\right)} \Delta_{B}(k)\left[\square+m^{2}-2 i k_{\mu} \partial^{\mu}-k^{2}\right. \\
& \left.-q F^{\mu \nu} x_{\nu} k_{\mu}-\frac{1}{4} q^{2} F^{\mu \alpha} F_{\mu \beta} x_{\alpha} x^{\beta}\right] \phi\left(x, x^{\prime}\right)=\delta^{4}\left(x-x^{\prime}\right) .
\end{aligned}
$$

We further substitute $\phi\left(x, x^{\prime}\right)$ from Eq. (8) into Eq. (9) and obtain

$$
\begin{gathered}
\int \frac{d^{4} k}{(2 \pi)^{4}} e^{-i k \cdot\left(x-x^{\prime}\right)} \Delta_{B}(k)\left[-k^{2}+m^{2}-q k^{\mu} F_{\mu \nu}\left(x-x^{\prime}\right)^{\nu}\right. \\
\left.-\frac{1}{4} q^{2} F^{\mu \alpha} F_{\mu \beta}\left(x-x^{\prime}\right)_{\alpha}\left(x-x^{\prime}\right)^{\beta}\right]=\delta^{4}\left(x-x^{\prime}\right),
\end{gathered}
$$

where we have used the fact that $\phi(x, x)=1$. This can be verified from Eq. (8) using the antisymmetric property of $F^{\mu \nu}$. Each term in Eq. (10) is now translation invariant and can be expressed as

$$
\begin{aligned}
& \int \frac{d^{4} k}{(2 \pi)^{4}} \Delta_{B}(k)\left[-k^{2}+m^{2}-i q k^{\mu} F_{\mu \nu} \tilde{\partial}^{\nu}\right. \\
& \left.+\frac{1}{4} q^{2} F^{\mu \alpha} F_{\mu \beta} \tilde{\partial}_{\alpha} \tilde{\partial}^{\beta}\right] e^{-i k \cdot\left(x-x^{\prime}\right)}=\delta^{4}\left(x-x^{\prime}\right),
\end{aligned}
$$

where we have used the notation $\tilde{\partial}_{\mu}=\left(\frac{\partial}{\partial k^{\mu}}\right)$. In order to extract $\Delta_{B}(k)$ from Eq. (11), it is necessary to swap the positions of $\Delta_{B}(k)$ and $e^{-i k \cdot\left(x-x^{\prime}\right)}$. This swapping is done at the cost of the addition of a term that contains a total fourmomentum derivative $\left(\tilde{\partial}_{\mu} J^{\mu}\right)$, i.e.,

$$
\begin{aligned}
& \int \frac{d^{4} k}{(2 \pi)^{4}} e^{-i k \cdot\left(x-x^{\prime}\right)}\left[-k^{2}+m^{2}+i q F_{\mu \nu} \tilde{\partial}^{\nu} k^{\mu}\right. \\
& \left.+\frac{1}{4} q^{2} F^{\mu \alpha} F_{\mu \beta} \tilde{\partial}_{\alpha} \tilde{\partial}^{\beta}\right] \Delta_{B}(k)+\int \frac{d^{4} k}{(2 \pi)^{4}} \tilde{\partial}_{\mu} J^{\mu}=\delta^{4}\left(x-x^{\prime}\right) .
\end{aligned}
$$

$J^{\mu}$ in the above equation contains the propagator $\Delta_{B}(k)$. Now the $d^{4} k$ integral of the second term on the lhs can be converted to a surface integral using Gauss' theorem, and if we assume that $D(k)$ is a well-behaved function this term will vanish. Thus, the momentum-space propagator $\Delta_{B}(k)$ satisfies the following differential equation:

$$
\left[-k^{2}+m^{2}+i q F_{\mu \nu} \tilde{\partial}^{\nu} k^{\mu}+\frac{1}{4} q^{2} F^{\mu \alpha} F_{\mu \beta} \tilde{\partial}_{\alpha} \tilde{\partial}^{\beta}\right] \Delta_{B}(k)=1 .
$$

Let us now consider a constant external magnetic field in the $+z$ direction, i.e., $\vec{B}=B \hat{z}$, which implies that the nonzero components of the electromagnetic field-strength tensor $F^{\mu \nu}$ are $F^{12}=-F^{21}$. So any four-vector $a^{\mu} \equiv$ $\left(a^{0}, a^{1}, a^{2}, a^{3}\right)$ is decomposed into $a^{\mu}=\left(a_{\|}^{\mu}+a_{\perp}^{\mu}\right)$, with $a_{\|}^{\mu} \equiv\left(a^{0}, 0,0, a^{3}\right)$ and $a_{\perp}^{\mu} \equiv\left(0, a^{1}, a^{2}, 0\right)$. The corresponding metric tensors are $g_{\|}^{\mu \nu}=\operatorname{diag}(1,0,0,-1)$ and $g_{\perp}^{\mu \nu}=$ $\operatorname{diag}(0,-1,-1,0)$, which satisfy $g^{\mu \nu}=\left(g_{\|}^{\mu \nu}+g_{\perp}^{\mu \nu}\right)$. Therefore, the propagator $\Delta_{B}(k)$ (being a Lorentz scalar) will be a function of $k_{\|}^{2}$ and $k_{\perp}^{2}$, i.e., $\Delta_{B}(k)=\Delta_{B}\left(k_{\|}^{2}, k_{\perp}^{2}\right)$. Hence, the third term in the square brackets on the lhs of Eq. (13) can be written as

$$
\begin{aligned}
& i q F_{\mu \nu} \tilde{\partial}^{\nu}\left[k^{\mu} \Delta_{B}\left(k_{\|}^{2}, k_{\perp}^{2}\right)\right] \\
& \quad=i q F_{\mu \nu}\left[g^{\mu \nu}+2 k^{\mu} k_{\|}^{\nu} \frac{\partial}{\partial k_{\|}^{2}}+2 k^{\mu} k_{\perp}^{\nu} \frac{\partial}{\partial k_{\perp}^{2}}\right] \Delta_{B}\left(k_{\|}^{2}, k_{\perp}^{2}\right)=0 .
\end{aligned}
$$


It is also trivial to check that

$$
F^{\mu \alpha} F_{\mu \beta} \tilde{\partial}_{\alpha} \tilde{\partial}^{\beta}=B^{2} g_{\perp}^{\alpha \beta} \tilde{\partial}_{\alpha} \tilde{\partial}_{\beta}=B^{2} \tilde{\square}_{\perp},
$$

where $\tilde{\square}_{\perp}=g_{\perp}^{\mu \nu} \tilde{\partial}_{\alpha} \tilde{\partial}_{\beta}$. Finally, Eq. (13) becomes

$$
\left[-k^{2}+m^{2}+\frac{1}{4}(q B)^{2} \tilde{\square}_{\perp}\right] \Delta_{B}(k)=1 .
$$

We now expand the propagator as a power series in $q B$,

$$
\Delta_{B}(k)=\sum_{i=0}^{\infty}(q B)^{i} \Delta_{i}(k)
$$

and substitute in Eq. (16) to obtain

$$
\sum_{i=0}^{\infty}\left[(q B)^{i}\left(-k^{2}+m^{2}\right)+(q B)^{i+2} \frac{1}{4} \tilde{\square}_{\perp}\right] \Delta_{i}(k)=1 .
$$

Equating the coefficients of different powers of $q B$ on both sides of the above equation, we get,

$$
\begin{aligned}
& \Delta_{0}(k)=\left(\frac{-1}{k^{2}-m^{2}+i \epsilon}\right), \\
& \Delta_{1}(k)=0, \\
& \Delta_{n}(k)=-\Delta_{0}(k) \frac{1}{4} \tilde{\square}_{\perp} \Delta_{n-2}(k) \text { for } n \geq 2 .
\end{aligned}
$$

Equation (19) is a recursion relation, and it immediately follows that $\Delta_{3}(k)=\Delta_{5}(k)=\Delta_{7}(k)=\cdots=0$. Using this relation, one can calculate the charged scalar propagator up to any order in $e B$. For example,

$$
\Delta_{2}(k)=-\Delta_{0}(k) \frac{1}{4} \tilde{\square}_{\perp} \Delta_{0}(k)=\left[\frac{k_{\|}^{2}-k_{\perp}^{2}-m^{2}}{\left(k^{2}-m^{2}+i \epsilon\right)^{4}}\right] .
$$

Therefore, the propagator becomes

$$
\begin{aligned}
\Delta_{B}(k)= & \Delta_{0}(k)+(q B)^{2} \Delta_{2}(k)+\mathcal{O}(q B)^{4} \\
= & \left(\frac{-1}{k^{2}-m^{2}+i \epsilon}\right)+(q B)^{2}\left[\frac{k_{\|}^{2}-k_{\perp}^{2}-m^{2}}{\left(k^{2}-m^{2}+i \epsilon\right)^{4}}\right] \\
& +\mathcal{O}(q B)^{4} .
\end{aligned}
$$

\section{FERMION PROPAGATOR IN AN EXTERNAL MAGNETIC FIELD}

Following a similar procedure as in the previous section, we find that the Dirac equation with an anomalous magnetic moment $(\kappa)$ in the momentum-space representation is given by $[47,48]$

$$
\left[\not p-\frac{i}{2} q F^{\mu \nu} \gamma_{\mu} \frac{\partial}{\partial p^{\nu}}-m_{f}-\frac{1}{2} \kappa \sigma \cdot F\right] S_{B}(p)=1 .
$$

To obtain the power expansion, we write

$$
S_{B}=S_{0}+S_{1},
$$

where $S_{0}$ represents the vacuum propagator and $S_{1}$ represents its linear-order correction in the presence of an external magnetic field. Now, let us define the operator

$$
\hat{O}=\left[\frac{i}{2} q F^{\mu \nu} \gamma_{\mu} \frac{\partial}{\partial p^{\nu}}+\frac{1}{2} \kappa \sigma \cdot F\right] .
$$

Using the perturbative expansion in the Dirac equation and neglecting the higher-order $\hat{O} S_{1}$ term, we obtain

$$
S_{1}=S_{0} \hat{O} S_{0} .
$$

Thus, the linear-order correction to the weak expansion of the propagator is nothing but an operator of noncommutative gamma matrices and differentials sandwiched between the familiar vacuum propagators. Following a similar strategy, one can extend the series to higher-order terms in powers of $B$. As we shall see, in our case the leading-order contribution of the external magnetic field is due to the quadratic correction of the weak-field propagator and not the simpler linear-order one. Thus, we must extend the perturbative series as

$$
S_{B}=S_{0}+S_{1}+S_{2},
$$

for which one obtains

$$
S_{2}=S_{0} \hat{O} S_{1}
$$

where $S_{1}=S_{0} \hat{O} S_{0}$ is given by (see Refs. [47,48])

$$
S_{1}=\frac{1}{\left(p^{2}-m_{f}^{2}+i \epsilon\right)^{2}} \times\left[q B \gamma_{5}\left[(p \cdot b) \not h-(p \cdot u) \not b+m_{f} \not \not \not b\right]+\kappa B\left[\left(\not p+m_{f}\right) \gamma_{5} \not \not \not h\left(\not p+m_{f}\right)\right]\right],
$$

with $u^{\mu} \equiv(1,0,0,0)$ and $b^{\mu} \equiv(0,0,0,1)$ in the fluid rest frame. It is straightforward to derive the expression for $S_{2}$, and after plugging in the correction terms we finally obtain the weak-field expansion of the fermion propagator:

$$
\begin{aligned}
& S_{B}(p, m)=\frac{-(\not p+m)}{p^{2}-m^{2}+i \epsilon}+(q B) \frac{i \gamma^{1} \gamma^{2}\left(\not \not_{\|}+m\right)}{\left(p^{2}-m^{2}+i \epsilon\right)^{2}}+(\kappa B) \frac{(\not p+m) i \gamma^{1} \gamma^{2}(\not p+m)}{\left(p^{2}-m^{2}+i \epsilon\right)^{2}}+(q B)^{2} \frac{-2\left\{p_{\perp}^{2}\left(\not \phi_{\|}+m\right)-\not \not_{\perp}\left(p_{\|}^{2}-m^{2}\right)\right\}}{\left(p^{2}-m^{2}+i \epsilon\right)^{4}}
\end{aligned}
$$

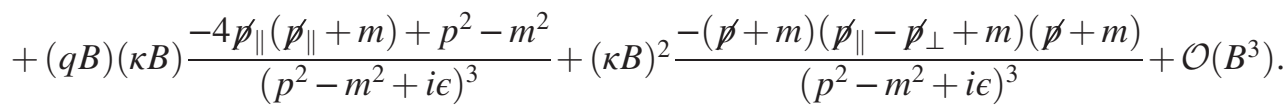


In order to express $S_{B}(p, m)$ in a more compact form, we use the procedure given in Ref. [49] and write

$$
\left(\frac{-1}{p^{2}-m^{2}+i \epsilon}\right)^{n}=\left.\hat{A}_{n-1} \Delta_{F}\left(p, m_{1}\right)\right|_{m_{1}=m},
$$

where

$$
\Delta_{F}(p, m)=\left(\frac{-1}{p^{2}-m^{2}+i \epsilon}\right)
$$

and

$$
\hat{A}_{n}=\frac{(-1)^{n}}{n !} \frac{\partial^{n}}{\partial\left(m_{1}^{2}\right)^{n}} .
$$

Using Eqs. (29)-(31), we can rewrite Eq. (28) as

$$
S_{B}(p, m)=\left.\hat{F}\left(p, m, m_{1}\right) \Delta_{F}\left(p, m_{1}\right)\right|_{m_{1}=m},
$$

where

$$
\begin{aligned}
& \hat{F}\left(p, m, m_{1}\right)=(\not p+m)+(q B) i \gamma^{1} \gamma^{2}(\not \not \|+m) \hat{A}_{1} \\
& +(\kappa B)(\not p+m) i \gamma^{1} \gamma^{2}(\not p+m) \hat{A}_{1} \\
& -2(q B)^{2}\left\{p_{\perp}^{2}\left(\not \not_{\|}+m\right)-\not p_{\perp}\left(p_{\|}^{2}-m^{2}\right)\right\} \hat{A}_{3} \\
& +(q B)(\kappa B)\left\{4 \not \not_{\|}\left(\not \not_{\|}+m\right)-p^{2}+m^{2}\right\} \hat{A}_{2} \\
& +(\kappa B)^{2}(\not p+m)\left(\not p \|-\not \not_{\perp}+m\right)(\not p+m) \hat{A}_{2} \\
& +\mathcal{O}\left(B^{3}\right) \text {. }
\end{aligned}
$$

We conclude this section by considering the fermion propagator at finite temperature and density along with the external magnetic field. For this, we use the real-time formalism of thermal field theory where the thermal propagator becomes a $2 \times 2$ matrix. However, it is sufficient to know the 11-component of the matrix propagator [50], which is given by [51]

$S_{11}(p, m)=S_{B}(p)-\eta(p \cdot u)\left[S_{B}(p)-\gamma^{0} S_{B}^{\dagger}(p) \gamma^{0}\right]$,

where

$\eta(p \cdot u)=\Theta(p \cdot u) f_{+}(p \cdot u)+\Theta(-p \cdot u) f_{-}(-p \cdot u)$

with

$$
f_{ \pm}(p \cdot u)=\left[\exp \left(\frac{p \cdot u \mp \mu}{T}\right)+1\right]^{-1} .
$$

Substituting Eq. (32) into Eq. (34) and using the fact that $\gamma^{0} \hat{F}^{\dagger}\left(p, m, m_{1}\right) \gamma^{0}=\hat{F}\left(p, m, m_{1}\right)$, we get

$$
\begin{aligned}
& S_{11}(p, m) \\
& \quad=\left.\hat{F}\left(p, m, m_{1}\right)\left[\Delta_{F}\left(p, m_{1}\right)-2 \pi i \eta(p \cdot u) \delta\left(p^{2}-m_{1}^{2}\right)\right]\right|_{m_{1}=m} .
\end{aligned}
$$

\section{EFFECTIVE MASS OF THE NUCLEON IN THE WALECKA MODEL}

The propagation of nucleons in hot and dense nuclear matter is well described using quantum hadrodynamics (QHD), details of which can be found in Refs. [52,53]. We briefly summarize the main formalism of QHD at zero magnetic field. We start with the real-time thermal propagator matrix of the nucleon [50,54],

$$
\boldsymbol{S}_{0}\left(p, m_{N}\right)=\left(\not p+m_{N}\right) \boldsymbol{V}\left[\begin{array}{cc}
\Delta_{F}\left(p, m_{N}\right) & 0 \\
0 & -\Delta_{F}^{*}\left(p, m_{N}\right)
\end{array}\right] \boldsymbol{V},
$$

where the diagonalizing matrix $\boldsymbol{V}$ is given by

$$
\boldsymbol{V}=\left[\begin{array}{cc}
N_{2} & -N_{1} e^{\beta \mu / 2} \\
N_{1} e^{-\beta \mu / 2} & N_{2}
\end{array}\right],
$$

with

$$
\begin{aligned}
N_{1}(p \cdot u)= & \sqrt{f_{+}(p \cdot u)} \Theta(p \cdot u)+\sqrt{f_{-}(-p \cdot u)} \Theta(-p \cdot u), \\
N_{2}(p \cdot u)= & \sqrt{1-f_{+}(p \cdot u)} \Theta(p \cdot u) \\
& +\sqrt{1-f_{-}(-p \cdot u)} \Theta(-p \cdot u) .
\end{aligned}
$$

In the Walecka model, the nucleons interact with the scalar meson $\sigma$ and vector meson $\omega$. The interaction Lagrangian is

$$
\mathcal{L}_{\mathrm{QHD}}=g_{\sigma N N} \bar{\Psi} \Psi \sigma-g_{\omega N N} \bar{\Psi} \gamma^{\mu} \Psi \omega_{\mu},
$$

where $\Psi=\left[\begin{array}{l}p \\ n\end{array}\right]$ is the nucleon isospin doublet and the values of the coupling constants are given by $g_{\sigma N N}=9.57$ and $g_{\omega N N}=11.67$ [52]. The complete nucleon propagator matrix $\boldsymbol{S}^{\prime}\left(p, m_{N}\right)$ in the presence of these interactions is obtained from the Dyson-Schwinger equation,

$$
S^{\prime}=S_{0}-S_{0} \Sigma S^{\prime}
$$

where $\boldsymbol{\Sigma}$ is the one-loop thermal self-energy matrix of the nucleon. It can be shown that [50] the complete propagator and self-energy matrices are diagonalized by $\boldsymbol{V}$ and $\boldsymbol{V}^{-1}$, respectively. This in turn diagonalizes the Dyson-Schwinger equation and Eq. (41) becomes an algebraic equation (in thermal space),

$$
\overline{S^{\prime}}=\bar{S}_{0}-\bar{S}_{0} \bar{\Sigma} \overline{S^{\prime}}
$$

We note that each term in the above equation is a $4 \times 4$ matrix in Dirac space. Here $\bar{S}_{0}\left(p, m_{N}\right)=-\left(\not p+m_{N}\right) \Delta_{F}\left(p, m_{N}\right)$, and $\bar{\Sigma}$ is the 11-component of the matrix $\boldsymbol{V}^{-1} \boldsymbol{\Sigma} \boldsymbol{V}^{-1}$ and is called the thermal self-energy function. In the Walecka model the Dirac structure of $\bar{\Sigma}$ is 


$$
\bar{\Sigma}=\left(\Sigma_{s} \mathbb{1}+\Sigma_{v}^{\mu} \gamma_{\mu}\right)=\left(\Sigma_{s} \mathbb{1}+\not{\Sigma}_{v}\right) .
$$

Using Eq. (43), we can solve Eq. (42) and obtain

$$
\overline{S^{\prime}}\left(p, m_{N}\right)=\left(\not P+m_{N}^{*}\right) \Delta_{F}\left(P, m_{N}^{*}\right),
$$

where

$$
P=\left(p-\Sigma_{v}\right) \quad \text { and } \quad m_{N}^{*}=\left(m+\Sigma_{s}\right) .
$$

We can finally write down the complete propagator matrix,

$$
\boldsymbol{S}^{\prime}\left(p, m_{N}\right)=\left(\not P+m_{N}^{*}\right) \boldsymbol{V}\left[\begin{array}{cc}
\Delta_{F}\left(P, m_{N}^{*}\right) & 0 \\
0 & -\Delta_{F}^{*}\left(P, m_{N}^{*}\right)
\end{array}\right] \boldsymbol{V},
$$

whose 11-component is

$$
\begin{aligned}
S_{11}^{\prime}\left(p, m_{N}\right)= & S_{F}\left(P, m_{N}^{*}\right) \\
& -\eta(P \cdot u)\left[S_{F}\left(P, m_{N}^{*}\right)-\gamma^{0} S_{F}^{\dagger}\left(P, m_{N}^{*}\right) \gamma^{0}\right] .
\end{aligned}
$$
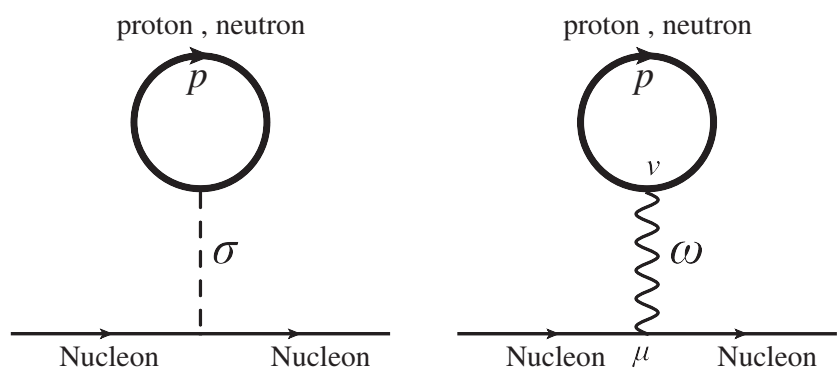

FIG. 1. Feynman diagrams for the one-loop self-energy of the nucleon in the Walecka model. Bold line indicates the complete/ dressed propagator.

Now we calculate the nucleon self-energy function $\bar{\Sigma}$ using the interaction Lagrangian given in Eq. (40) and consider only the tadpole Feynman diagrams, as shown in Fig. 1. We note that the loop particles are dressed, i.e., the propagator for the loop particles is $\boldsymbol{S}^{\prime}\left(p, m_{N}\right)$, as given in Eq. (46). Applying Feynman rules to Fig. 1, we obtain the 11-component of the thermal self-energy as

$$
\begin{aligned}
\Sigma_{11}= & -\left(\frac{g_{\sigma N N}^{2}}{m_{\sigma}^{2}}\right) i \int \frac{d^{4} p}{(2 \pi)^{4}} \operatorname{Tr}\left[{S^{\prime}}_{11}^{(\mathrm{p})}\left(p, m_{N}\right)+{S^{\prime}}_{11}^{(\mathrm{n})}\left(p, m_{N}\right)\right] \\
& +\gamma_{\mu}\left(\frac{g_{\omega N N}^{2}}{m_{\omega}^{2}}\right) i \int \frac{d^{4} p}{(2 \pi)^{4}} \operatorname{Tr}\left[\gamma^{\mu}{S^{\prime}}_{11}^{(\mathrm{p})}\left(p, m_{N}\right)+\gamma^{\mu}{S^{\prime}}_{11}^{(\mathrm{n})}\left(p, m_{N}\right)\right],
\end{aligned}
$$

where the superscripts (p) and (n) correspond to the proton and neutron, respectively. It is easy to show that $\operatorname{Re} \Sigma_{11}=\operatorname{Re} \bar{\Sigma}$. Thus, from Eq. (43) we get

$$
\begin{gathered}
\operatorname{Re} \Sigma_{s}=-\left(\frac{g_{\sigma N N}^{2}}{m_{\sigma}^{2}}\right) \operatorname{Re} i \int \frac{d^{4} p}{(2 \pi)^{4}} \operatorname{Tr}\left[{S^{\prime}}_{11}^{(\mathrm{p})}\left(p, m_{N}\right)+{S^{\prime}}_{11}^{(\mathrm{n})}\left(p, m_{N}\right)\right], \\
\operatorname{Re} \Sigma_{v}^{\mu}=\left(\frac{g_{\omega N N}^{2}}{m_{\omega}^{2}}\right) \operatorname{Re} i \int \frac{d^{4} p}{(2 \pi)^{4}} \operatorname{Tr}\left[\gamma^{\mu}{S^{\prime}}_{11}^{(\mathrm{p})}\left(p, m_{N}\right)+\gamma^{\mu}{S^{\prime}}_{11}^{(\mathrm{n})}\left(p, m_{N}\right)\right] .
\end{gathered}
$$

Substituting $S_{11}^{\prime}\left(p, m_{N}\right)$ from Eq. (47) into Eqs. (49) and (50) and performing the $d p^{0}$ integral, we get

$$
\begin{gathered}
\operatorname{Re} \Sigma_{s}\left(m_{N}^{*}\right)= \\
\operatorname{Re} \Sigma_{s}^{\text {(pure vacuum) }}-\left(\frac{4 g_{\sigma N N}^{2} m_{N}^{*}}{m_{\sigma}^{2}}\right) \int \frac{d^{3} p}{(2 \pi)^{3}}\left(\frac{1}{\Omega_{p}}\right)\left[N_{+}^{p}+N_{-}^{p}\right], \\
\operatorname{Re} \Sigma_{v}^{\mu}\left(m_{N}^{*}\right)=\left(\frac{4 g_{\omega N N}^{2}}{m_{\omega}^{2}}\right) \int \frac{d^{3} p}{(2 \pi)^{3}}\left[N_{+}^{p}-N_{-}^{p}\right] \delta_{0}^{\mu},
\end{gathered}
$$

where $\Omega_{p}=\sqrt{\vec{p}^{2}+\left(m_{N}^{*}\right)^{2}}$ and

$$
N_{ \pm}^{p}=\left[\exp \left(\frac{\Omega_{p} \mp \mu}{T}\right)+1\right]^{-1}
$$

In Eq. (51), $\operatorname{Re} \Sigma_{s}^{\text {(pure vacuum) }}$ is given by

$$
\operatorname{Re} \Sigma_{s}^{\text {(pure vacuum) }}=\left(\frac{8 m_{N}^{*} g_{\sigma N N}^{2}}{m_{\sigma}^{2}}\right) \operatorname{Re} i \int \frac{d^{4} p}{(2 \pi)^{4}}\left[\frac{1}{p^{2}-\left(m_{N}^{*}\right)^{2}+i \epsilon}\right] .
$$

We will neglect the contribution of the vacuum self-energy term $\operatorname{Re} \Sigma_{s}^{\text {(pure vacuum) }}$ in Eq. (51), following the mean-field theory (MFT) [52] approach. 
The effective mass of the nucleon $\left(m_{N}^{*}\right)$ can be calculated from the pole of the complete nucleon propagator, which essentially involves solving the self-consistent equation

$$
m_{N}^{*}=m_{N}+\operatorname{Re} \Sigma_{s}\left(m_{N}^{*}\right)
$$

Let us now turn to the external magnetic field. Since we are only interested in the effective mass of the nucleon, let us calculate the scalar self-energy $\operatorname{Re} \Sigma_{s}\left(m_{N}^{*}\right)$. In this case, the proton and neutron propagators in Eq. (49) have to be replaced by $S_{11}^{\prime}\left(p, m_{N}\right) \rightarrow S_{11}\left(P, m_{N}^{*}\right)$, where $S_{11}(p, m)$ is defined in Eq. (37). This implies

$$
{S^{\prime}}_{11}^{(\mathrm{p}, \mathrm{n})}\left(p, m_{N}\right)=\left.\hat{F}^{(\mathrm{p}, \mathrm{n})}\left(P, m_{N}^{*}, m_{1}\right)\left[\Delta_{F}\left(P, m_{1}\right)-2 \pi i \eta(P \cdot u) \delta\left(P^{2}-m_{1}^{2}\right)\right]\right|_{m_{1}=m_{N}^{*}},
$$

where $\hat{F}^{(\mathrm{p})}\left(p, m, m_{1}\right)$ and $\hat{F}^{(\mathrm{n})}\left(p, m, m_{1}\right)$ are obtained from Eq. (33) by replacing $q$ and $\kappa$ with the corresponding values for the proton and neutron, respectively, i.e., for the proton $q \rightarrow|e|, \kappa \rightarrow \kappa_{\mathrm{p}}$, and for the neutron $q \rightarrow 0, \kappa \rightarrow \kappa_{\mathrm{n}}$. Here $|e|$ is the absolute electronic charge and the anomalous magnetic moments of the proton and neutron are given by $\kappa_{\mathrm{p}}=g_{\mathrm{p}}\left(\frac{|e|}{2 m_{N}}\right)$ and $\kappa_{\mathrm{n}}=g_{\mathrm{n}}\left(\frac{|e|}{2 m_{N}}\right)$, respectively, with $g_{\mathrm{p}}=1.79$ and $g_{\mathrm{n}}=-1.91$.

Substituting Eq. (56) into Eq. (49) and shifting the momentum $p \rightarrow\left(p+\Sigma_{V}\right)$, we get

$$
\begin{gathered}
\operatorname{Re} \Sigma_{s}=-\left.\left(\frac{g_{\sigma N N}^{2}}{m_{\sigma}^{2}}\right) \operatorname{Re} i \int \frac{d^{4} p}{(2 \pi)^{4}} \operatorname{Tr}\left[\hat{F}^{(\mathrm{p})}\left(p, m_{N}^{*}, m_{1}\right)+\hat{F}^{(\mathrm{n})}\left(p, m_{N}^{*}, m_{1}\right)\right] \Delta_{F}\left(p, m_{1}\right)\right|_{m_{1}=m_{N}^{*}} \\
-\left.\left(\frac{g_{\sigma N N}^{2}}{m_{\sigma}^{2}}\right) \int \frac{d^{4} p}{(2 \pi)^{4}} \operatorname{Tr}\left[\hat{F}^{(\mathrm{p})}\left(p, m_{N}^{*}, m_{1}\right)+\hat{F}^{(\mathrm{n})}\left(p, m_{N}^{*}, m_{1}\right)\right] 2 \pi \eta(p \cdot u) \delta\left(p^{2}-m_{1}^{2}\right)\right|_{m_{1}=m_{N}^{*}} \\
\Rightarrow \operatorname{Re} \Sigma_{s}=\Sigma_{s}^{(\text {vacuum })}+\Sigma_{s}^{(\text {medium })},
\end{gathered}
$$

where

$$
\begin{gathered}
\Sigma_{s}^{\text {(vacuum })}=-\left.\left(\frac{g_{\sigma N N}^{2}}{m_{\sigma}^{2}}\right) \operatorname{Re} i \int \frac{d^{4} p}{(2 \pi)^{4}} \hat{T}\left(p, m_{N}^{*}, m_{1}\right) \Delta_{F}\left(p, m_{1}\right)\right|_{m_{1}=m_{N}^{*}}, \\
\Sigma_{s}^{(\text {medium })}=-\left.\left(\frac{g_{\sigma N N}^{2}}{m_{\sigma}^{2}}\right) \int \frac{d^{4} p}{(2 \pi)^{4}} \hat{T}\left(p, m_{N}^{*}, m_{1}\right) 2 \pi \eta(p \cdot u) \delta\left(p^{2}-m_{1}^{2}\right)\right|_{m_{1}=m_{N}^{*}} .
\end{gathered}
$$

In the above equations

$$
\begin{aligned}
\hat{T}\left(p, m_{N}^{*}, m_{1}\right)= & \operatorname{Tr}\left[\hat{F}^{(\mathrm{p})}\left(p, m_{N}^{*}, m_{1}\right)+\hat{F}^{(\mathrm{n})}\left(p, m_{N}^{*}, m_{1}\right)\right] \\
= & 8 m_{N}^{*}-8 m_{N}^{*}(e B)^{2} p_{\perp}^{2} \hat{A}_{3}+4 m_{N}^{*}\left\{\left(\kappa_{\mathrm{p}} B\right)^{2}+\left(\kappa_{\mathrm{n}} B\right)^{2}\right\}\left\{\left(m_{N}^{*}\right)^{2}+p^{2}-2 p_{\perp}^{2}+2 p_{\|}^{2}\right\} \hat{A}_{2} \\
& +4(|e| B)\left(\kappa_{\mathrm{p}} B\right)\left\{\left(m_{N}^{*}\right)^{2}-p^{2}+4 p_{\|}^{2}\right\} \hat{A}_{2} .
\end{aligned}
$$

The detailed calculations of $\Sigma_{s}^{(\text {vacuum })}$ and $\Sigma_{s}^{\text {(medium) }}$ are provided in Appendices A and B. The expression for $\Sigma_{s}^{\text {(vacuum) }}$ can be read from Eq. (A10) as

$$
\Sigma_{s}^{(\text {vacuum })}=\left(\frac{g_{\sigma N N}^{2}}{4 \pi^{2} m_{\sigma}^{2}}\right)\left[\frac{(e B)^{2}}{3 m_{N}^{*}}+\left\{\left(\kappa_{\mathrm{p}} B\right)^{2} m_{N}^{*}+\left(\kappa_{\mathrm{n}} B\right)^{2} m_{N}^{*}+(|e| B)\left(\kappa_{\mathrm{p}} B\right)\right\}\left\{\frac{1}{2}+2 \ln \left(\frac{m_{N}^{*}}{m_{N}}\right)\right\}\right] .
$$

The calculation of $\Sigma_{s}^{\text {(medium) }}$ is performed separately for two different cases: the zero-temperature case and the finitetemperature case. For zero temperature, from Eq. (B12) we have

$$
\begin{aligned}
\Sigma_{s}^{(\text {medium })}= & -\left(\frac{2 g_{\sigma N N}^{2}}{\pi^{2} m_{\sigma}^{2}}\right)\left[m_{N}^{*} I_{2}\left(\mu_{\mathrm{B}}, m_{N}^{*}\right)+\frac{1}{3}(e B)^{2} m_{N}^{*} C_{1}\left(\mu_{\mathrm{B}}, m_{N}^{*}\right)\right. \\
& \left.+2\left\{m_{N}^{*}\left(\kappa_{\mathrm{p}} B\right)^{2}+m_{N}^{*}\left(\kappa_{\mathrm{n}} B\right)^{2}+(|e| B)\left(\kappa_{\mathrm{p}} B\right)\right\}\left\{m_{N}^{* 2} C_{1}\left(\mu_{\mathrm{B}}, m_{N}^{*}\right)+\frac{1}{3} C_{2}\left(\mu_{\mathrm{B}}, m_{N}^{*}\right)\right\}\right] .
\end{aligned}
$$


For finite temperature, from Eq. (B16) we have

$$
\begin{aligned}
\Sigma_{s}^{(\text {medium })}= & -\left(\frac{2 g_{\sigma N N}^{2}}{\pi^{2} m_{\sigma}^{2}}\right) \int_{0}^{\infty}|\vec{p}|^{2} d|\vec{p}|\left[m_{N}^{*}\left(\tilde{C}_{1}^{+p}+\tilde{C}_{1}^{-p}\right)+\frac{2}{3} m_{N}^{*}(e B)^{2}|\vec{p}|^{2}\left(\tilde{C}_{3}^{+p}+\tilde{C}_{3}^{-p}\right)\right. \\
& \left.+2\left(m_{N}^{* 2}+\frac{2}{3}|\vec{p}|^{2}\right)\left\{m_{N}^{*}\left(\kappa_{\mathrm{p}} B\right)^{2}+m_{N}^{*}\left(\kappa_{\mathrm{n}} B\right)^{2}+(|e| B)\left(\kappa_{\mathrm{p}} B\right)\right\}\left(\tilde{C}_{2}^{+p}+\tilde{C}_{2}^{-p}\right)\right] .
\end{aligned}
$$

The definitions of the functions $I_{2}, C_{1}, C_{2}, \tilde{C}_{1}^{ \pm}, \tilde{C}_{2}^{ \pm}$, and $\tilde{C}_{3}^{ \pm}$ can be found in Appendix B.

\section{NUMERICAL RESULTS}

We begin this section by obtaining the effective nucleon mass with an external magnetic field at zero temperature and zero density. In this case the contribution from $\Sigma_{s}^{\text {(medium) }}=0$. Thus, we need to solve the transcendental equation

$$
m_{N}^{*}=m_{N}+\Sigma_{s}^{(\text {vacuum })}\left(m_{N}^{*}\right)
$$

where $\Sigma_{s}^{(\mathrm{vacuum})}\left(m_{N}^{*}\right)$ is given in Eq. (62). At first we neglect the effect of the anomalous magnetic moments of nucleons so that the above equation simplifies to

$$
m_{N}^{*}=m_{N}+\frac{g_{\sigma N N}^{2}(e B)^{2}}{12 \pi^{2} m_{\sigma}^{2} m_{N}^{*}}
$$

which can be solved analytically to obtain

$$
m_{N}^{*}=\frac{1}{2}\left[m_{N}+\sqrt{m_{N}^{2}+\frac{g_{\sigma N N}^{2}(e B)^{2}}{3 \pi^{2} m_{\sigma}^{2}}}\right] .
$$

As can be seen from the above equation, the effective nucleon mass increases monotonically with the increase of $e B$. This enhancement is shown in Fig. 2, where it is also compared with the result from Ref. [33]. Though the

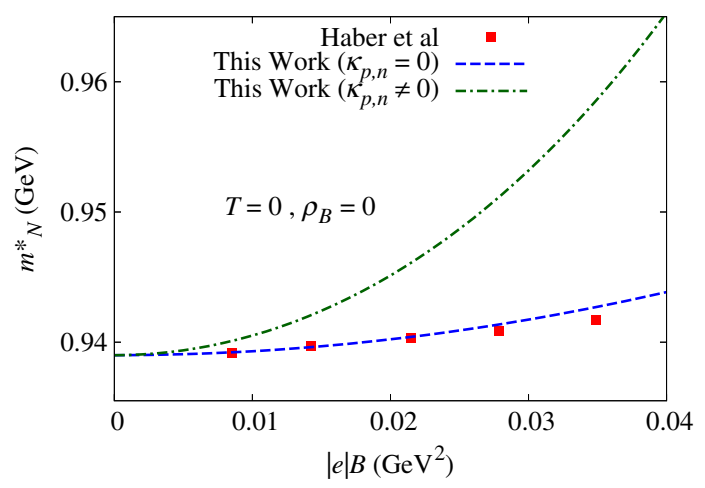

FIG. 2. Variation of $m_{N}^{*}$ with $|e| B$ at zero temperature and zero density. Results with and without the anomalous magnetic moments of nucleons are compared with results from Ref. [33]. current approach to obtain the effective nucleon mass differs from Ref. [33], there exists a noticeable quantitative agreement between the two results in the weak magnetic field regime. Now we include the anomalous magnetic moments of nucleons and solve Eq. (65) numerically. We find that the incorporation of the nucleon magnetic moment further increases the effective mass and this effect remains significant even in the case of weak magnetic fields, as shown in Fig. 2. In other words, the nucleon magnetic moments favor the magnetic catalysis effect at zero temperature and zero baryon density.

We now study the nucleon effective mass in the presence of an external magnetic field at finite baryon density and zero temperature. As can be seen from Eqs. (62) and (63), the scalar self-energy $\Sigma_{s}$ is a function of the magnetic field $B$ and baryon chemical potential $\mu_{\mathrm{B}}$ of the medium. It is customary to use the total baryon density $\rho_{\mathrm{B}}$ instead of $\mu_{\mathrm{B}}$, where

$$
\begin{aligned}
\rho_{\mathrm{B}} & =4 \int \frac{d^{3} p}{(2 \pi)^{3}} \Theta\left(\mu_{\mathrm{B}}-\sqrt{|\vec{p}|^{2}+m_{N}^{* 2}}\right) \\
& =\left(\frac{2}{3 \pi^{2}}\right)\left[\mu_{\mathrm{B}}^{2}-m_{N}^{* 2}\right]^{3 / 2} .
\end{aligned}
$$

Inverting the above equation, we get the baryon chemical potential in terms of the baryon density as

$$
\mu_{\mathrm{B}}=\sqrt{\left(\frac{3 \pi^{2}}{2} \rho_{\mathrm{B}}\right)^{2 / 3}+m_{N}^{* 2}} .
$$

We have expressed the strength of the magnetic field $B$ with respect to the pion mass scale $\left(B_{\pi}\right)$, defined as

$$
|e| B_{\pi}=m_{\pi}^{2}=0.0196 \mathrm{GeV}^{2} .
$$

Similarly, the total baryon density $\rho_{B}$ is expressed with respect to the normal nuclear matter density $\rho_{0}=0.16 \mathrm{fm}^{-3}$.

Since we will be solving the transcendental equation (55), we first plot $m_{N}^{*}-\operatorname{Re} \Sigma_{s}\left(m_{N}^{*}\right)$ as a function of $m_{N}^{*}$ in Fig. 3 . Figure 3(a) depicts the variation of this quantity at three different values of magnetic field $\left(B / B_{\pi}=0,1\right.$ and 2$)$ with baryon density $\rho_{B}=2 \rho_{0}$, whereas Fig. 3(b) shows its variation at three different values of total baryon density $\left(\rho_{B} / \rho_{0}=1,2\right.$ and 3$)$ with magnetic field $B=B_{\pi}$. The intersections of these graphs with the horizontal line 

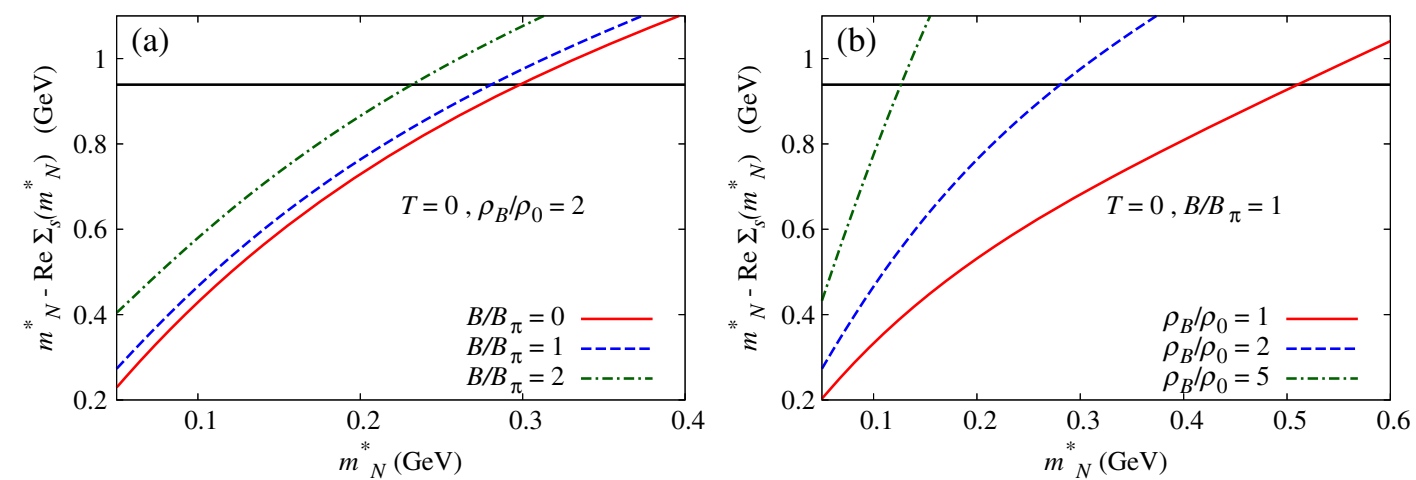

FIG. 3. Variation of $m_{N}^{*}-\operatorname{Re} \Sigma_{s}\left(m_{N}^{*}\right)$ with $m_{N}^{*}$ at zero temperature for (a) three different values of the magnetic field $\left(B=0, B_{\pi}, 2 B_{\pi}\right)$ at baryon density $\rho_{\mathrm{B}}=2 \rho_{0}$, and (b) three different values of the baryon density $\left(\rho_{B}=\rho_{0}, 2 \rho_{0}, 5 \rho_{0}\right)$ at magnetic field $\left(B=B_{\pi}\right)$. Here $|e| B_{\pi}=m_{\pi}^{2}=0.0196 \mathrm{GeV}^{2}$ and $\rho_{0}=0.16 \mathrm{fm}^{-3}$. The horizontal black solid line corresponds to $m_{N}^{*}=m_{N}=939 \mathrm{MeV}$.

corresponding to $m_{N}^{*}=m_{N}=939 \mathrm{MeV}$ represent the solutions of Eq. (55). We notice from these figures that $\operatorname{Re} \Sigma\left(m_{N}^{*}\right)$ is always less than zero and it monotonically decreases as we increase $m_{N}^{*}$. Also, for a particular value of $m_{N}^{*}, \operatorname{Re} \Sigma\left(m_{N}^{*}\right)$ decreases with the increase of $B$ and $\rho_{B}$. In Fig. 4(a), the variation of the effective nucleon mass with baryon density is shown at three different values of magnetic field $\left(B=0, B_{\pi}, 2 B_{\pi}\right)$. As can be seen from the figure, $m_{N}^{*} / m_{N}$ decreases with the increase of $\rho_{\mathrm{B}}$ and becomes less than 0.5 at $\rho_{\mathrm{B}}=2 \rho_{0}$. It can be checked that the contribution from the first term in the square brackets in
Eq. (63) plays the dominant role in determining $\rho_{\mathrm{B}}$ as well as the $e B$ dependences of the effective mass, whereas the net contribution from all other terms in $\Sigma_{s}^{(\text {medium })}$ and $\Sigma_{s}^{(\text {vacuum })}$ [see Eq. (62)] remains subleading throughout. Also, it is clear from Fig. 4(a) that, with the increase of $|e| B$, the effective mass decreases and the effect of the external magnetic field is greater in the lower $\rho_{\mathrm{B}}$ region. At very high $\rho_{\mathrm{B}}\left(\gtrsim 5 \rho_{0}\right)$ it is expected that the effect of $|e| B$ on the nucleon effective mass will be negligible. However, the conclusions based on the weak-field approximation are not
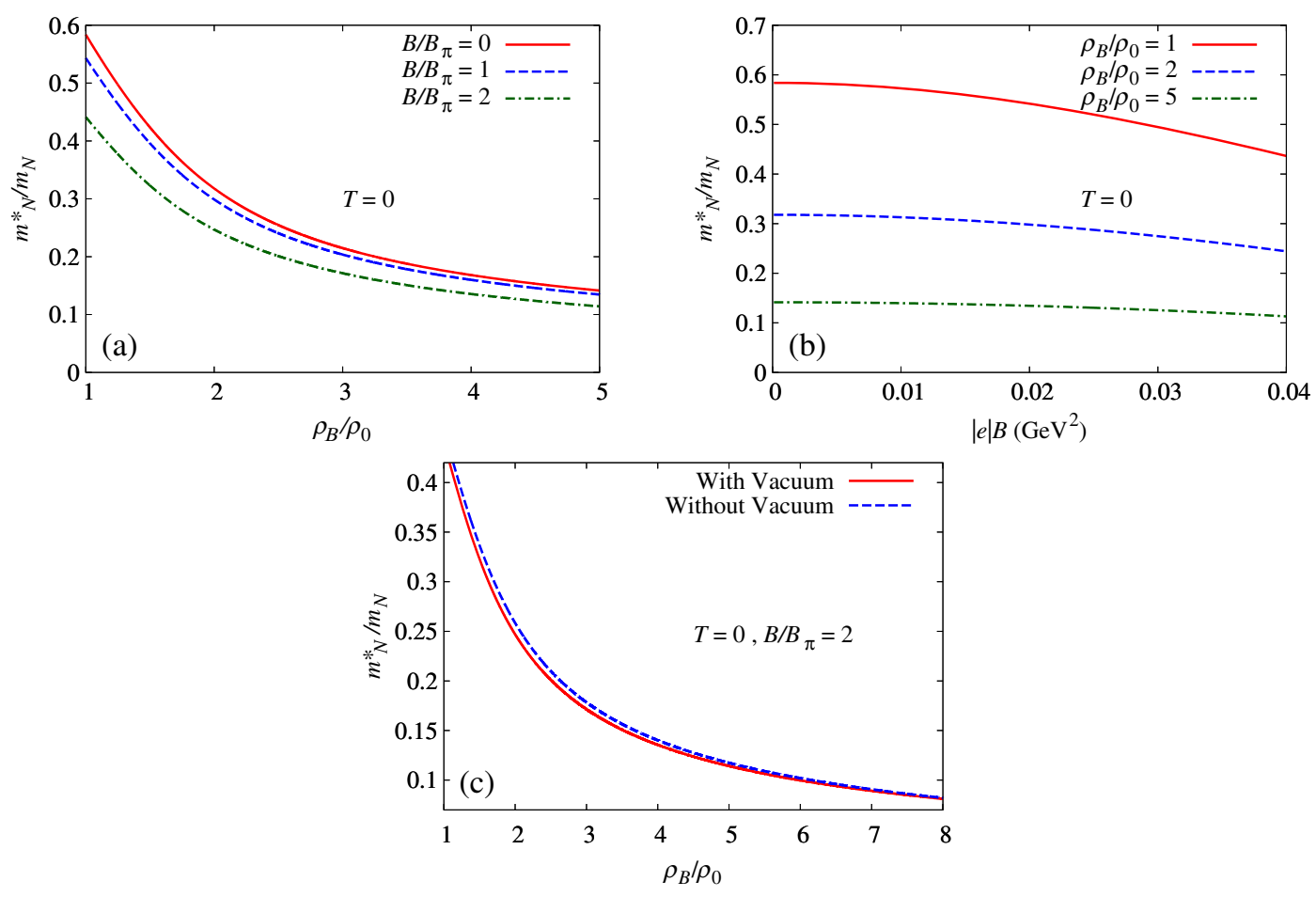

FIG. 4. Variation of the effective mass of the nucleon at zero temperature (a) with baryon density for three different values of magnetic field $\left(B=0, B_{\pi}, 2 B_{\pi}\right)$, and (b) with magnetic field for three different values of baryon density $\left(\rho_{B}=\rho_{0}, 2 \rho_{0}, 5 \rho_{0}\right)$. Here $|e| B_{\pi}=$ $m_{\pi}^{2}=0.0196 \mathrm{GeV}^{2}$ and $\rho_{0}=0.16 \mathrm{fm}^{-3}$. (c) At $B=2 B_{\pi}$, the variation of the effective nucleon mass with baryon density is compared with the case where the vacuum contribution is ignored. 
reliable for arbitrarily large or small densities, as will be discussed later.

In Fig. 4(b), the variation of $m_{N}^{*} / m_{N}$ with $|e| B$ is shown at three different values of baryon density $\left(\rho_{B}=\rho_{0}, 2 \rho_{0}\right.$, $\left.5 \rho_{0}\right)$. We find a small decrease in the effective nucleon mass with $|e| B$. In order to observe the effect of the vacuum selfenergy correction to the effective mass of the nucleon, we compare the density variations of $m_{N}^{*}$ with and without the vacuum contribution, as shown in Fig. 4(c). Here the external magnetic field is kept fixed at $B=2 B_{\pi}$. We note that the effect of the vacuum correction is subleading with respect to the medium contribution at nonzero baryon density, and the correction to $m_{N}^{*}$ due to the vacuum self-energy remains less than $6 \%$. It is also interesting to observe the relative effect of the external magnetic field on the effective nucleon mass, as shown in Fig. 5, where the ratio $m_{N}^{*}(e B) / m_{N}^{*}(e B=0)$ is plotted as a function of $e B$ at three different baryon densities $\left(\rho_{B}=\rho_{0}, 2 \rho_{0}, 5 \rho_{0}\right)$. We note that $m_{N}^{*}$ decreases by about $25 \%$ at a magnetic field $e B \sim 0.04 \mathrm{GeV}^{2}$. The inset plot shows the lower $e B$ region up to $e B=0.01 \mathrm{GeV}^{2}$, which corresponds to the typical values of magnetic field expected inside a neutron star/magnetar. At the maximum value $e B=0.01 \mathrm{GeV}^{2}$, the effective mass of the nucleon decreases by less than $2 \%$.

Until now we have considered that under the weakfield approximation the modifications from the nonvanishing anomalous magnetic moment arise only through the effective mass. Moreover, we assumed that the modification to the expression for the proton density as a summation over Landau levels can also be ignored for weak external fields. The motivation behind this approximation lies in the fact that with smaller values of external field, the Landau levels become more closely spaced, giving rise to a continuum at $e B \rightarrow 0$. In that

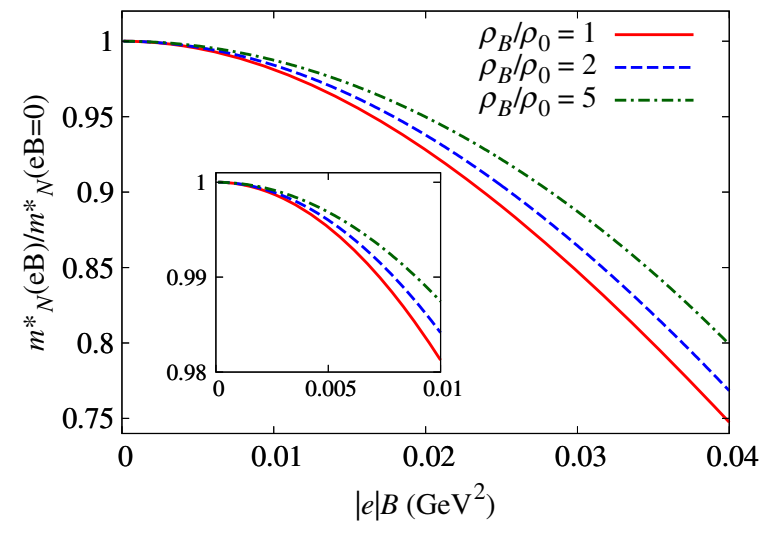

FIG. 5. At $T=0$, the ratio of the effective mass $m^{*}$ at nonzero $e B$ and at zero $e B$ is plotted as a function of $e B$ for three different values of baryon density $\left(\rho_{B}=\rho_{0}, 2 \rho_{0}, 5 \rho_{0}\right)$. The inset plot shows the low- $e B$ region up to $e B=0.01 \mathrm{GeV}^{2}$ relevant for neutron stars/magnetars. Here $\rho_{0}=0.16 \mathrm{fm}^{-3}$.

case, the summations that appear due to the Landau quantization can be replaced by the corresponding momentum integrals, giving rise to the same expression for the proton and neutron densities in isospin-symmetric matter. As a result, the expression for the baryon density as given in Eq. (68) is still valid even in the presence of $e B$ as long as the external fields are sufficiently weak to make the summation-to-integral conversion plausible. It is advantageous to use this approximate expression to obtain the effective mass of the nucleons as, in this case, $\mu_{\mathrm{B}}$ can be analytically expressed in terms of $\rho_{\mathrm{B}}$, which simplifies the numerics. However, to check the validity of the approximations, it is reasonable to incorporate these magnetic modifications into the expression for the net baryon density, which now becomes $[55,56]$

$$
\begin{aligned}
\rho_{\mathrm{B}}= & \sum_{s \in\{ \pm 1\}} \int \frac{d^{3} p}{(2 \pi)^{3}} \Theta\left\{\mu_{\mathrm{B}}-\sqrt{p_{z}^{2}+\left(\sqrt{m_{N}^{* 2}-p_{\perp}^{2}}-s \kappa_{\mathrm{n}} B\right)^{2}}\right\} \\
& +\frac{e B}{(2 \pi)^{2}} \sum_{s \in\{ \pm 1\}} \sum_{n=0}^{\infty}\left(1-\delta_{0}^{n} \delta_{-1}^{s}\right) \int_{-\infty}^{\infty} d p_{z} \Theta\left\{\mu_{\mathrm{B}}-\sqrt{p_{z}^{2}+\left(\sqrt{m_{N}^{* 2}+2 n|e| B}-s \kappa_{\mathrm{p}} B\right)^{2}}\right\} .
\end{aligned}
$$

Performing the momentum integral in the above equation, we obtain

$$
\begin{aligned}
\rho_{\mathrm{B}}= & \sum_{s \in\{ \pm 1\}} \frac{1}{12 \pi^{2}}\left[3 \pi \mu_{\mathrm{B}}^{2} s \kappa_{\mathrm{n}} B+2 \sqrt{\mu_{\mathrm{B}}^{2}-\left(m_{N}^{*}-s \kappa_{\mathrm{n}} B\right)^{2}}\left\{2 \mu_{\mathrm{B}}^{2}-2 m_{N}^{* 2}+m_{N}^{*} s \kappa_{\mathrm{n}} B+\left(s \kappa_{\mathrm{n}} B\right)^{2}\right\}\right. \\
& \left.+6 \mu_{\mathrm{B}}^{2} s \kappa_{\mathrm{n}} B \tan ^{-1}\left\{\frac{s \kappa_{\mathrm{n}} B-m_{N}^{*}}{\sqrt{\mu_{\mathrm{B}}^{2}-\left(m_{N}^{*}-s \kappa_{\mathrm{n}} B\right)^{2}}}\right\}\right]+\frac{e B}{2 \pi^{2}} \sum_{s \in\{ \pm 1\}} \sum_{n=0}^{n_{\max }}\left(1-\delta_{0}^{n} \delta_{-1}^{s}\right) \sqrt{\mu_{\mathrm{B}}^{2}-\left(\sqrt{m_{N}^{* 2}+2 n|e| B}-s \kappa_{\mathrm{p}} B\right)^{2}},
\end{aligned}
$$



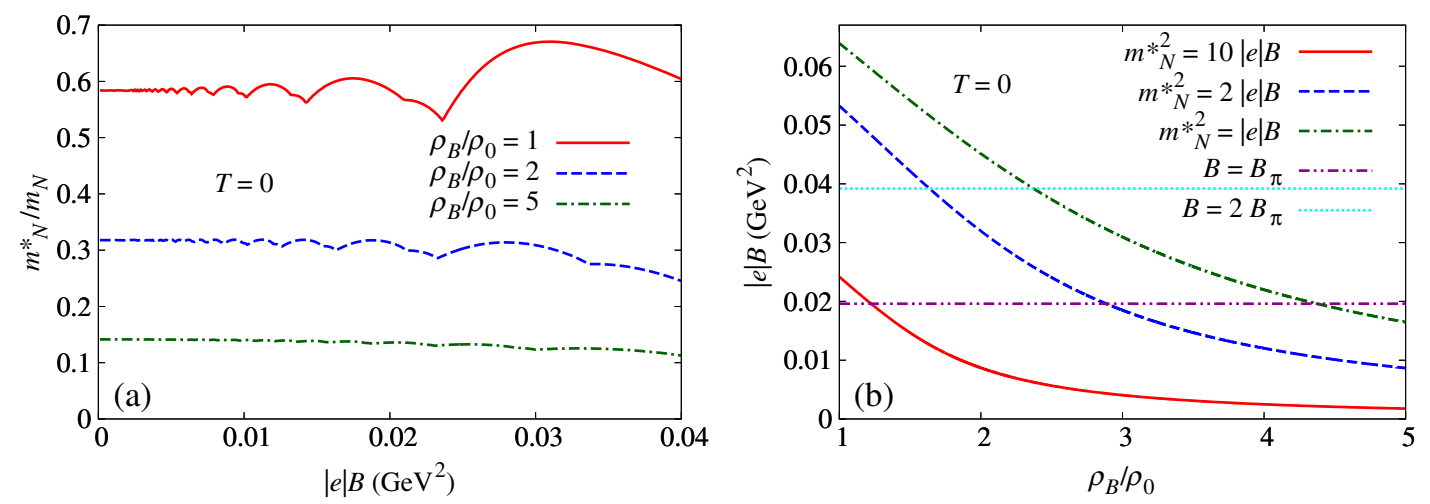

FIG. 6. Variation of the effective mass of the nucleon at zero temperature (a) with baryon density for three different values of magnetic field $\left(B=0, B_{\pi}, 2 B_{\pi}\right)$, and (b) with magnetic field for three different values of baryon density $\left(\rho_{B}=\rho_{0}, 2 \rho_{0}, 5 \rho_{0}\right)$. Here $|e| B_{\pi}=$ $m_{\pi}^{2}=0.0196 \mathrm{GeV}^{2}$ and $\rho_{0}=0.16 \mathrm{fm}^{-3}$.

where $n_{\max }=\left[\frac{\left(\mu_{\mathrm{B}}+s \kappa_{\mathrm{B}} B\right)^{2}-m_{N}^{* 2}}{2|e| B}\right]$ in which $[x]=$ the greatest integer less than or equal to $x$. The above equation cannot be analytically inverted in order to express $\mu_{B}$ as a function of $\rho_{B}$, which was possible for the $e B=0$ case [see Eq. (69)]. Thus we invert the equation numerically to obtain $\mu_{B}=\mu_{B}\left(\rho_{B}, e B\right)$. Using the above modified $\rho_{B}$, in Fig. 6 we replot the variation of the effective mass with the external field for the same set of densities $\rho_{B}=\rho_{0}, 2 \rho_{0}$, and
$5 \rho_{0}$. The oscillating behavior is consistent with Ref. [33]. Comparison with Fig. 4(b) suggests that the usual baryon density expression agrees reasonably well with the average qualitative behavior even in the presence of an external magnetic field as long as the background field strength is small, and the agreement is more pronounced for higher densities. However, going to arbitrarily large densities is restricted by the assumption of the weak-field expansion of the propagator which demands that the external $e B$ be
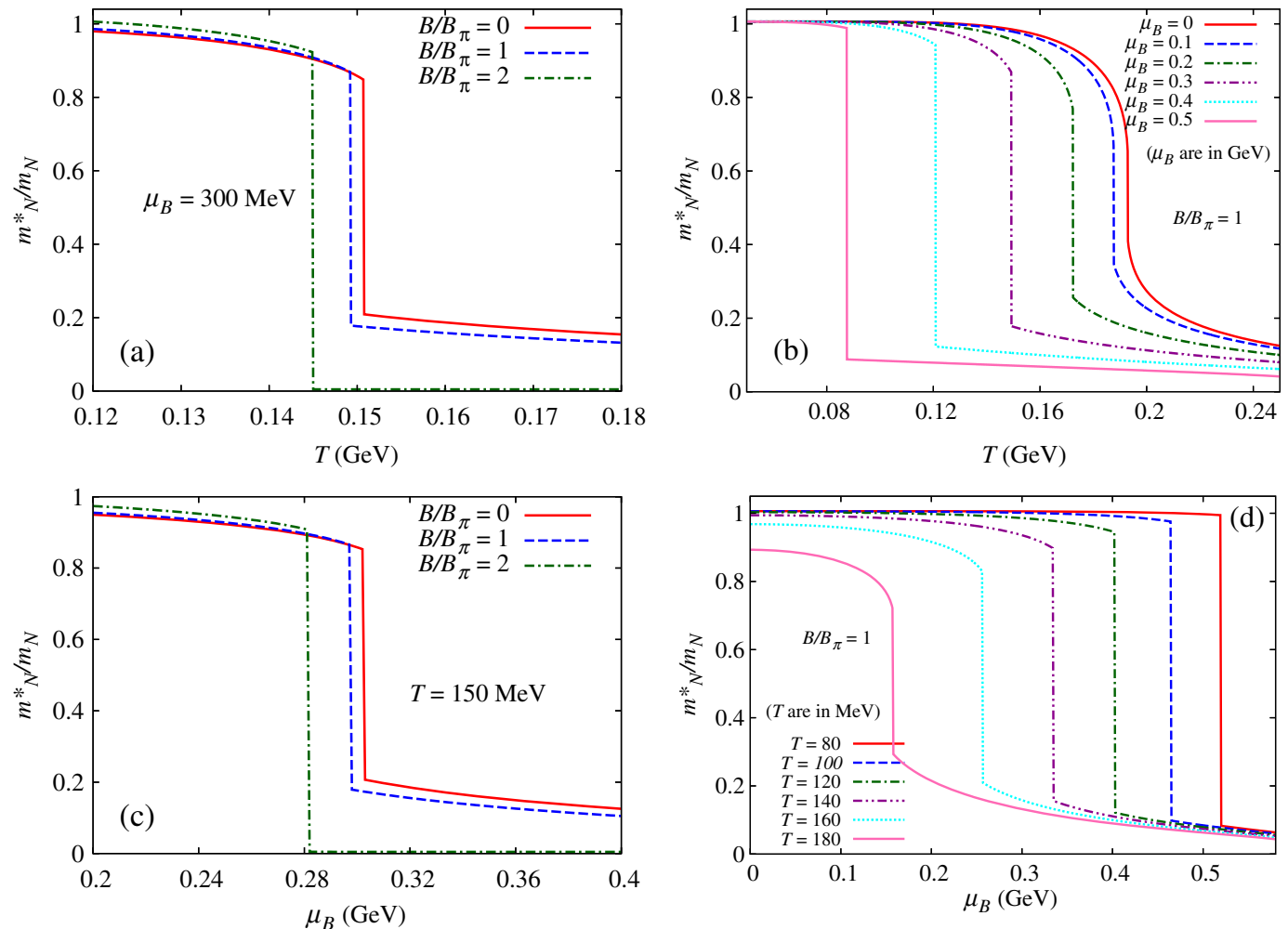

FIG. 7. Variation of the effective mass of the nucleon with temperature (a) at $\mu_{\mathrm{B}}=300 \mathrm{MeV}$ for three different values $B$ (0, $B_{\pi}$, and $\left.2 B_{\pi}\right)$, and (b) at $B=B_{\pi}$ for six different values of $\mu_{\mathrm{B}}(0,100,200,300,400$, and $500 \mathrm{MeV})$. We also show the variation of the effective mass of the nucleon with baryon chemical potential (c) at $T=150 \mathrm{MeV}$ for three different values of magnetic field $\left(B=0, B_{\pi}, 2 B_{\pi}\right)$, and (d) at $B=B_{\pi}$ for six different value of $T(80,100,120,140,160$, and $180 \mathrm{MeV})$. Here $|e| B_{\pi}=m_{\pi}^{2}=0.0196 \mathrm{GeV}^{2}$. 


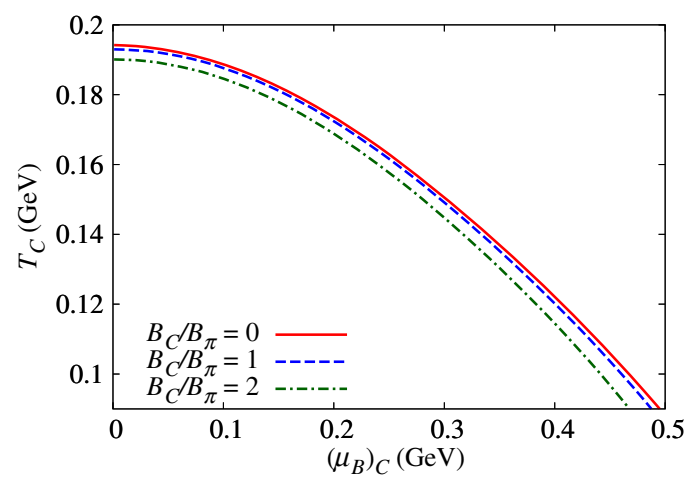

FIG. 8. Phase diagram for the vacuum to nuclear medium phase transition in the Walecka model for three different values of $B(0$, $B_{\pi}$, and $\left.2 B_{\pi}\right)$.

much smaller than $m_{N}^{* 2}$. Now, in addition to the external magnetic field this effective mass also depends on the density and, more importantly, it is a decreasing dependence. Thus, even if one starts with a constant $e B$ much lower than $m_{N}^{* 2}$, the decreasing trend of $m_{N}^{*}$ with density invalidates this basic weak-field assumption at some higher $\rho_{\mathrm{B}}$ value for which $m_{N}^{* 2}$ becomes comparable with the constant $e B$ used. To estimate this density value, we fix the maximum possible value of $e B$ that we consider as a fraction times $m_{N}^{* 2}$, where the fraction is chosen to be 0.5 or 0.1 . The corresponding variations with respect to $\rho_{\mathrm{B}}$ are shown in Fig. 6(b), where the case $e B=m_{N}^{* 2}$ is also plotted for comparison. Each of these curves in fact plays the role of a boundary, and for a given value of $\rho_{\mathrm{B}}$ only those $e B$ values that lie below them are allowed. The horizontal lines denote the constant magnetic field values used in this work. It is clear from the figure that, once we have chosen the maximum $e B$ curve (say, the $e B=0.5 m_{N}^{* 2}$ curve), its intersection with each horizontal line provides the maximum density (i.e., around $3 \rho_{0}$ for $B=B_{\pi}$ and around $1.8 \rho_{0}$ for $B=2 B_{\pi}$ ) up to which the $e B$ value corresponding to that line can be considered as "weak."

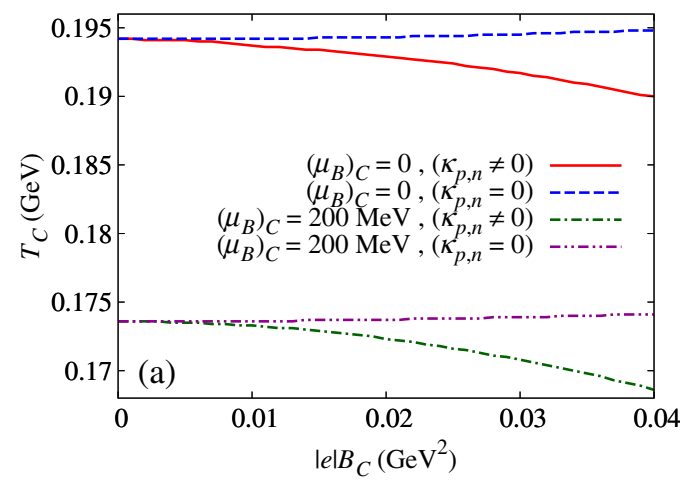

We now study the variation of $m_{N}^{*} / m_{N}$ with temperature and baryon chemical potential in Fig. 7. Figure 7(a) depicts the variation of $m_{N}^{*} / m_{N}$ with $T$ at $\mu_{\mathrm{B}}=300 \mathrm{MeV}$ and at three different values $B\left(0, B_{\pi}\right.$, and $\left.2 B_{\pi}\right)$, whereas Fig. 7(b) shows its variation at $B=B_{\pi}$ and at six different values $\mu_{\mathrm{B}}$ $(0,100,200,300,400$, and $500 \mathrm{MeV})$. As can be seen from the figure, the effective nucleon mass suffers a sudden decrease at a particular temperature corresponding to the vacuum to nuclear medium phase transition $[33,52]$. We call this transition temperature $T_{C}$ and we calculate it numerically from the slope of these plots. As can be seen from Fig. 7(a), $T_{C}$ decreases with the increase of $B$, which may be identified as IMC in the Walecka model. In Fig. 7(b), we observe that $T_{C}$ decreases with the increase of $\mu_{\mathrm{B}}$. The corresponding variation of $m_{N}^{*} / m_{N}$ with $\mu_{\mathrm{B}}$ is shown in Figs. 7(b) and 7(c). Analogous to the upper panels, we see a phase transition at a particular $\mu_{\mathrm{B}}$ and we call this transition chemical potential $\left(\mu_{\mathrm{B}}\right)_{C}$. As can be seen in the graphs, $\left(\mu_{\mathrm{B}}\right)_{C}$ decreases with the increase in $B$ and $T$.

The behavior of $T_{C}$ and $\left(\mu_{\mathrm{B}}\right)_{C}$ at different $B$ can be seen in Fig. 8, where we show the phase diagram for the vacuum to nuclear medium phase transition at three different values of $B\left(0, B_{\pi}\right.$, and $\left.2 B_{\pi}\right)$. With the increase in $\left(\mu_{\mathrm{B}}\right)_{C}, T_{C}$ decreases and vice versa. Also, with the increase in $B$ the phase boundary in this $T-\mu_{\mathrm{B}}$ plane moves towards lower values of $T$ and $\mu_{\mathrm{B}}$, thus exhibiting IMC.

We conclude this section by plotting the variation of $T_{C}$ and $\left(\mu_{\mathrm{B}}\right)_{C}$ with external magnetic field in Fig. 9. Figure 9(a) shows the variation of $T_{C}$ with $|e| B$ at two different values of $\mu_{\mathrm{B}}(0$ and $200 \mathrm{MeV})$, whereas Fig. 9(b) shows the corresponding variation at two different values of $T(100$ and $130 \mathrm{MeV})$. As already discussed, both $T_{C}$ and $\left(\mu_{\mathrm{B}}\right)_{C}$ decrease with the increase in $B$, which is characteristic of the IMC effect. However, once the anomalous magnetic moment is ignored, $T_{C}$ as well as $\left(\mu_{\mathrm{B}}\right)_{C}$ can be observed to slowly increase with the external magnetic field, thus exhibiting MC as expected [33].

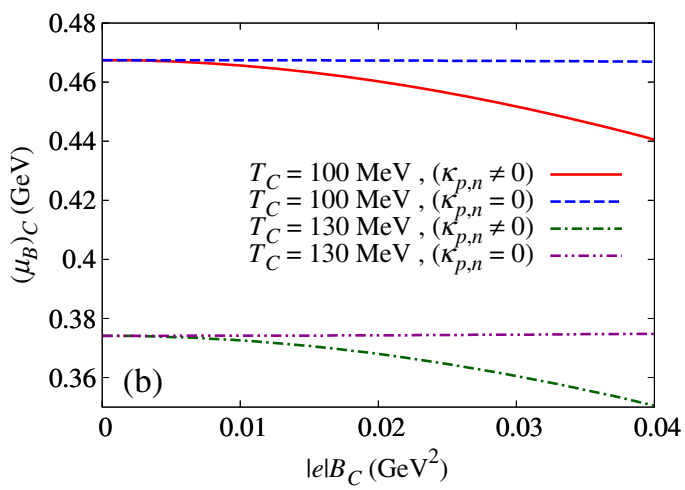

FIG. 9. (a) Variation of the transition temperature with magnetic field at two different values of $\mu_{\mathrm{B}}(0$ and $200 \mathrm{MeV})$. (b) Variation of the transition baryon chemical potential with magnetic field at two different values of $T$ (100 and $130 \mathrm{MeV})$. Cases with and without the AMM of nucleons are shown separately. 


\section{SUMMARY}

In this article we have used the Walecka model to study the vacuum to nuclear matter phase transition in the presence of a weak and constant background magnetic field within the mean-field approximation. In the case of a weak magnetic field, the nucleon propagators were derived as a series in powers of $q B$ and $\kappa B$, where $q$ and $\kappa$ represent the charge and anomalous magnetic moment of the nucleon. The effective mass of the nucleon $\left(m_{N}^{*}\right)$ was obtained from the pole of the nucleon propagator selfconsistently. At zero temperature and zero density, the incorporation of the anomalous magnetic moment was shown to favor the effective mass enhancement with the external magnetic field. The functional dependence of $m_{N}^{*}$ on the background field was extended to the case of nonzero nuclear density and further extended to the finite-temperature regime. It was observed that in the case of vanishing temperature within a dense nuclear medium the effective mass decreases with the background magnetic field, and this trend was shown to survive in the case of nonzero temperature as well. Moreover, there exists a particular temperature (denoted by $T_{C}$ in the text) for which the effective nucleon mass suffers a sudden decrease corresponding to the vacuum to nuclear medium phase transition. It has been shown that this critical temperature decreases with the increase of $B$ which can be identified as inverse magnetic catalysis in the Walecka model, whereas the opposite behavior is obtained in the case of a vanishing magnetic moment. Thus, it can be inferred that in the presence of an external magnetic field the anomalous magnetic moment of the nucleon plays a crucial role in characterizing the nature of the vacuum to nuclear matter transition at finite temperature and density. It should be mentioned here that Haber et al. [33] had speculated that the incorporation of an anomalous magnetic moment could counteract the effect of magnetic catalysis [40]. Our study not only supports this speculation, but also concludes that the effect is significant enough to alter the qualitative behavior of the nucleon effective mass even in the weak magnetic field regime. However, it should be noted here that the weak-field approximation actually restricts the regime of validity of the present study, as discussed in detail in the text. The maximum value of the external magnetic field used in the present study was $0.04 \mathrm{GeV}^{2}$, which was considered "weak" only up to a density of $1.8 \rho_{0}$ where the assumption of "weakness" is fixed by the condition that the chosen external field has to remain less than $50 \%$ of the effective mass. We also note that in the case of the Walecka model, MC or IMC can only be seen indirectly. Similar studies in the extended linear sigma model might be interesting, as in that case the possibility of (approximate) chiral symmetry restoration is incorporated into the model framework. However, we should also mention that in the case of zero magnetic moment only the quantitative difference in the behavior of the effective mass can be attributed to the presence of the chiral partners [33], whereas the qualitative behavior that was the main interest throughout this article seems to show model independence. Before applying the present result to obtain the characteristics of compact stars (such as the mass-radius relationship or the equation of state), beta equilibrium and charge neutrality conditions have to be properly incorporated, which lies beyond the scope of the present study.

\section{ACKNOWLEDGMENTS}

S. G. acknowledges the Center for Nuclear Theory, Variable Energy Cyclotron Centre, and the Indian Institute of Technology Gandhinagar for support.

\section{APPENDIX A: CALCULATION OF $\boldsymbol{\Sigma}_{s}^{(\text {vacuum })}$}

From Eq. (59) we have

$$
\Sigma_{s}^{(\text {vacuum })}=\left.\left(\frac{g_{\sigma N N}^{2}}{m_{\sigma}^{2}}\right) \operatorname{Re} i \int \frac{d^{d} p}{(2 \pi)^{d}} \hat{T}\left(p, m_{N}^{*}, m_{1}\right) \frac{1}{p^{2}-m_{1}^{2}+i \epsilon}\right|_{m_{1}=m_{N}^{*}, d \rightarrow 4} .
$$

In order to perform the $d^{4} p$ integration, we use the following identities [57]:

$$
\begin{aligned}
& \int \frac{d^{d} p}{(2 \pi)^{d}}\left(\frac{1}{p^{2}-\Delta}\right)=\frac{-i}{(4 \pi)^{d / 2}} \Gamma\left(1-\frac{d}{2}\right)\left(\frac{1}{\Delta}\right)^{1-d / 2} \\
& \int \frac{d^{d} p}{(2 \pi)^{d}}\left(\frac{p_{\perp}^{2}}{p^{2}-\Delta}\right)=\frac{i}{(4 \pi)^{d / 2}}\left(\frac{d}{4}\right) \Gamma\left(-\frac{d}{2}\right)\left(\frac{1}{\Delta}\right)^{-d / 2} \\
& \int \frac{d^{d} p}{(2 \pi)^{d}}\left(\frac{p_{\|}^{2}}{p^{2}-\Delta}\right)=\frac{i}{(4 \pi)^{d / 2}}\left(\frac{d}{4}\right) \Gamma\left(-\frac{d}{2}\right)\left(\frac{1}{\Delta}\right)^{-d / 2}
\end{aligned}
$$




$$
\int \frac{d^{d} p}{(2 \pi)^{d}}\left(\frac{p^{2}}{p^{2}-\Delta}\right)=\frac{i}{(4 \pi)^{d / 2}}\left(\frac{d}{2}\right) \Gamma\left(-\frac{d}{2}\right)\left(\frac{1}{\Delta}\right)^{-d / 2}
$$

Thus, Eq. (A1) becomes

$$
\Sigma_{s}^{(\text {vacuum })}=\operatorname{Re} \Sigma_{s}^{(\text {pure vacuum })}+\Sigma_{s}^{(\text {divergent })}+\Sigma_{s}^{(\text {regular })}
$$

where $\operatorname{Re} \Sigma_{s}^{\text {(pure vacuum) }}$ is the ultraviolet-divergent pure vacuum contribution given in Eq. (54), and

$$
\begin{gathered}
\Sigma_{s}^{(\text {divergent })}=-\left.\left(\frac{g_{\sigma N N}^{2}}{4 \pi^{2} m_{\sigma}^{2}}\right)\left\{\left(\kappa_{\mathrm{p}} B\right)^{2} m_{N}^{*}+\left(\kappa_{\mathrm{n}} B\right)^{2} m_{N}^{*}+(|e| B)\left(\kappa_{\mathrm{p}} B\right)\right\} \Gamma\left(2-\frac{d}{2}\right)\left(\frac{1}{m_{N}^{* 2}}\right)^{2-d / 2}\right|_{d \rightarrow 4} \\
\Sigma_{s}^{(\text {regular })}=\left(\frac{g_{\sigma N N}^{2}}{4 \pi^{2} m_{\sigma}^{2}}\right)\left[\frac{(e B)^{2}}{3 m_{N}^{*}}+\frac{1}{2}\left\{\left(\kappa_{\mathrm{p}} B\right)^{2} m_{N}^{*}+\left(\kappa_{\mathrm{n}} B\right)^{2} m_{N}^{*}+(|e| B)\left(\kappa_{\mathrm{p}} B\right)\right\}\right]
\end{gathered}
$$

Also in this case, we will neglect the pure vacuum contribution $\operatorname{Re} \Sigma_{s}^{\text {(pure vacuum) }}$ which is equivalent to using MFT. We now extract the divergence of $\Sigma_{s}^{\text {(divergent) }}$ from the pole of the gamma function and use the $\overline{\mathrm{MS}}$ scheme to obtain

$$
\Sigma_{s}^{(\text {divergent })}=\left(\frac{g_{\sigma N N}^{2}}{4 \pi^{2} m_{\sigma}^{2}}\right)\left\{\left(\kappa_{\mathrm{p}} B\right)^{2} m_{N}^{*}+\left(\kappa_{\mathrm{n}} B\right)^{2} m_{N}^{*}+(|e| B)\left(\kappa_{\mathrm{p}} B\right)\right\} \ln \left(\frac{m_{N}^{* 2}}{\Lambda}\right),
$$

where $\Lambda$ is a scale of dimension $\mathrm{GeV}^{2}$. Its value is fixed from the condition $\Sigma_{s}^{(\text {divergent })}\left(m_{N}^{*}=m_{N}\right)=0$, which gives $\Lambda=m_{N}^{2}$. So the final expression of $\Sigma_{s}^{(\text {vacuum })}$ becomes

$$
\Sigma_{s}^{(\text {vacuum })}=\left(\frac{g_{\sigma N N}^{2}}{4 \pi^{2} m_{\sigma}^{2}}\right)\left[\frac{(e B)^{2}}{3 m_{N}^{*}}+\left\{\left(\kappa_{\mathrm{p}} B\right)^{2} m_{N}^{*}+\left(\kappa_{\mathrm{n}} B\right)^{2} m_{N}^{*}+(|e| B)\left(\kappa_{\mathrm{p}} B\right)\right\}\left\{\frac{1}{2}+2 \ln \left(\frac{m_{N}^{*}}{m_{N}}\right)\right\}\right]
$$

\section{APPENDIX B: CALCULATION OF $\Sigma_{s}^{(\text {medium })}$}

From Eq. (60) we have

$$
\Sigma_{s}^{(\text {medium })}=-\left.\left(\frac{g_{\sigma N N}^{2}}{m_{\sigma}^{2}}\right) \int \frac{d^{4} p}{(2 \pi)^{4}} \hat{T}\left(p, m_{N}^{*}, m_{1}\right) 2 \pi \eta(p \cdot u) \delta\left(p^{2}-m_{1}^{2}\right)\right|_{m_{1}=m_{N}^{*}}
$$

where $\hat{T}\left(p, m_{N}^{*}, m_{1}\right)$ is given in Eq. (61). Using Eqs. (35) and (36), we can write the above equation as

$$
\Sigma_{s}^{(\text {medium })}=-\left(\frac{g_{\sigma N N}^{2}}{m_{\sigma}^{2}}\right) \int \frac{d^{3} p}{(2 \pi)^{3}} \int_{-\infty}^{+\infty} d p^{0} \hat{T}\left(p^{0}, \vec{p}, m_{N}^{*}, m_{1}\right)\left(\frac{1}{2 \omega_{1}}\right) \times\left[f_{+}\left(\omega_{1}\right) \delta\left(p^{0}-\omega_{1}\right)+f_{-}\left(\omega_{1}\right) \delta\left(p^{0}+\omega_{1}\right)\right]_{m_{1}=m_{N}^{*}}
$$

where $\omega_{1}=\sqrt{\vec{p}^{2}+m_{1}^{2}}$. Performing the $d p^{0}$ integration using the Dirac delta functions and noting that $\hat{T}\left(p^{0}, \vec{p}, m_{N}^{*}, m_{1}\right)$ is an even function of $p^{0}$, we get

$$
\Sigma_{s}^{(\text {medium })}=-\left(\frac{g_{\sigma N N}^{2}}{2 m_{\sigma}^{2}}\right) \int \frac{d^{3} p}{(2 \pi)^{3}} \hat{T}\left(p^{0}=\Omega_{p}, \vec{p}, m_{N}^{*}, m_{1}\right)\left(\frac{1}{\omega_{1}}\right) \times\left[f_{+}\left(\omega_{1}\right)+f_{-}\left(\omega_{1}\right)\right]_{m_{1}=m_{N}^{*}} .
$$

Substituting Eq. (61) into Eq. (B2) and performing the angular integration, we get

$$
\Sigma_{s}^{(\text {medium })}=-\left(\frac{g_{\sigma N N}^{2}}{8 \pi^{2} m_{\sigma}^{2}}\right) \int_{0}^{\infty}|\vec{p}|^{2} d|\vec{p}| \hat{B}\left(\vec{p}, m_{N}^{*}, m_{1}\right)\left(\frac{1}{\omega_{1}}\right) \times\left[f_{+}\left(\omega_{1}\right)+f_{-}\left(\omega_{1}\right)\right]_{m_{1}=m_{N}^{*}},
$$

where 


$$
\hat{B}\left(\vec{p}, m_{N}^{*}, m_{1}\right)=16 m_{N}^{*}+\frac{32}{3}(e B)^{2} m_{N}^{*}|\vec{p}|^{2} \hat{A}_{3}+16\left(2 m_{N}^{* 2}+\frac{4}{3}|\vec{p}|^{2}\right) \times\left\{m_{N}^{*}\left(\kappa_{\mathrm{p}} B\right)^{2}+m_{N}^{*}\left(\kappa_{\mathrm{n}} B\right)^{2}+(|e| B)\left(\kappa_{\mathrm{p}} B\right)\right\} \hat{A}_{2} .
$$

\section{Zero-temperature case}

From Eq. (36), at $T=0$ we have

$$
\lim _{T \rightarrow 0} f_{ \pm}\left(\omega_{1}\right)=\Theta\left( \pm \mu_{\mathrm{B}}-\omega_{1}\right)
$$

where $\mu_{\mathrm{B}}$ is the baryon chemical potential of the medium. Substituting Eq. (B5) into Eq. (B3), we get

$$
\Sigma_{s}^{(\text {medium })}=-\left.\left(\frac{g_{\sigma N N}^{2}}{8 \pi^{2} m_{\sigma}^{2}}\right) \int_{0}^{\infty}|\vec{p}|^{2} d|\vec{p}| \hat{B}\left(\vec{p}, m_{N}^{*}, m_{1}\right) \frac{1}{\omega_{1}} \Theta\left(\mu_{\mathrm{B}}-\omega_{1}\right)\right|_{m_{1}=m_{N}^{*}} .
$$

The $d|\vec{p}|$ integration of the above equation can be evaluated analytically using the following identities:

$$
\begin{gathered}
I_{2}(\mu, m)=\int_{0}^{\sqrt{\mu^{2}-m^{2}}} \frac{|\vec{p}|^{2} d|\vec{p}|}{\sqrt{|\vec{p}|^{2}+m^{2}}}=\frac{1}{2}\left[\mu \sqrt{\mu^{2}-m^{2}}+m^{2} \ln \left\{\frac{m}{\mu+\sqrt{\mu^{2}-m^{2}}}\right\}\right], \\
I_{4}(\mu, m)=\int_{0}^{\sqrt{\mu^{2}-m^{2}}} \frac{|\vec{p}|^{4} d|\vec{p}|}{\sqrt{|\vec{p}|^{2}+m^{2}}}=\frac{1}{8}\left[\mu\left(2 \mu^{2}-5 m^{2}\right) \sqrt{\mu^{2}-m^{2}}-3 m^{4} \ln \left\{\frac{m}{\mu+\sqrt{\mu^{2}-m^{2}}}\right\}\right] .
\end{gathered}
$$

Thus, we get

$$
\begin{aligned}
\Sigma_{s}^{(\text {medium })}= & -\left(\frac{2 g_{\sigma N N}^{2}}{\pi^{2} m_{\sigma}^{2}}\right)\left[m_{N}^{*} I_{2}\left(\mu_{\mathrm{B}}, m_{1}\right)+\frac{2}{3}(e B)^{2} m_{N}^{*} \hat{A}_{3} I_{4}\left(\mu_{\mathrm{B}}, m_{1}\right)\right. \\
& \left.+2\left\{m_{N}^{*}\left(\kappa_{\mathrm{p}} B\right)^{2}+m_{N}^{*}\left(\kappa_{\mathrm{n}} B\right)^{2}+(|e| B)\left(\kappa_{\mathrm{p}} B\right)\right\}\left\{m_{N}^{* 2} \hat{A}_{2} I_{2}\left(\mu_{\mathrm{B}}, m_{1}\right)+\frac{1}{3} \hat{A}_{2} I_{4}\left(\mu_{\mathrm{B}}, m_{1}\right)\right\}\right]_{m_{1}=m_{N}^{*}}
\end{aligned}
$$

It is now trivial to check that

$$
\begin{gathered}
\left.\hat{A}_{2} I_{2}\left(\mu, m_{1}\right)\right|_{m_{1}=m_{N}^{*}}=\left.2 \hat{A}_{3} I_{4}\left(\mu, m_{1}\right)\right|_{m_{1}=m_{N}^{*}}=\frac{\mu}{8 m_{N}^{* 2} \sqrt{\mu^{2}-m_{N}^{* 2}}}=C_{1}\left(\mu, m_{N}^{*}\right) \quad(\text { say }), \\
\left.\hat{A}_{2} I_{4}\left(\mu, m_{1}\right)\right|_{m_{1}=m_{N}^{*}}=-\left(\frac{3}{8}\right) \ln \left\{\frac{m_{N}^{*}}{\mu+\sqrt{\mu^{2}-m_{N}^{* 2}}}\right\}=C_{2}\left(\mu, m_{N}^{*}\right) \quad \text { (say). }
\end{gathered}
$$

Finally, $\Sigma_{s}^{(\text {medium })}$ becomes

$$
\begin{aligned}
\Sigma_{s}^{(\text {medium })}= & -\left(\frac{2 g_{\sigma N N}^{2}}{\pi^{2} m_{\sigma}^{2}}\right)\left[m_{N}^{*} I_{2}\left(\mu_{\mathrm{B}}, m_{N}^{*}\right)+\frac{1}{3}(e B)^{2} m_{N}^{*} C_{1}\left(\mu_{\mathrm{B}}, m_{N}^{*}\right)\right. \\
& \left.+2\left\{m_{N}^{*}\left(\kappa_{\mathrm{p}} B\right)^{2}+m_{N}^{*}\left(\kappa_{\mathrm{n}} B\right)^{2}+(|e| B)\left(\kappa_{\mathrm{p}} B\right)\right\}\left\{m_{N}^{* 2} C_{1}\left(\mu_{\mathrm{B}}, m_{N}^{*}\right)+\frac{1}{3} C_{2}\left(\mu_{\mathrm{B}}, m_{N}^{*}\right)\right\}\right] .
\end{aligned}
$$

\section{Finite-temperature case}

At finite temperature, the $d|\vec{p}|$ integration in Eq. (B3) cannot be performed analytically. We simplify the expression by evaluating the derivatives with respect to $m_{1}^{2}$ explicitly. For this, we use the following results:

$$
\left[\frac{f_{ \pm}\left(\omega_{1}\right)}{\omega_{1}}\right]_{m_{1}=m_{N}^{*}}=\frac{N_{ \pm}^{p}}{\Omega_{p}}=\tilde{C}_{1}^{ \pm p} \quad(\text { say })
$$




$$
\begin{gathered}
\hat{A}_{2}\left[\frac{f_{ \pm}\left(\omega_{1}\right)}{\omega_{1}}\right]_{m_{1}=m_{N}^{*}}=\frac{N_{ \pm}^{p}}{8 \Omega_{p}^{5}}\left[3+3\left(1-N_{ \pm}^{p}\right) \beta \Omega_{p}+\left\{1-3 N_{ \pm}^{p}+2\left(N_{ \pm}^{p}\right)^{2}\right\} \beta^{2} \Omega_{p}^{2}\right]=\tilde{C}_{2}^{ \pm p} \quad \text { (say) } \\
\hat{A}_{3}\left[\frac{f_{ \pm}\left(\omega_{1}\right)}{\omega_{1}}\right]_{m_{1}=m_{N}^{*}}= \\
\left.+\left\{1-7 N_{ \pm}^{p}+12\left(N_{ \pm}^{p}\right)^{2}-6\left(N_{ \pm}^{p}\right)^{3}\right\} \beta^{3} \Omega_{p}^{3}\right]=\tilde{C}_{3}^{ \pm p} \quad \text { (say) } \\
+\left\{15+15\left(1-N_{ \pm}^{p}\right) \beta \Omega_{p}+6\left\{1-3 N_{ \pm}^{p}+2\left(N_{ \pm}^{p}\right)^{2}\right\} \beta^{2} \Omega_{p}^{2}\right.
\end{gathered}
$$

From Eq. (B3) we obtain

$$
\begin{aligned}
\Sigma_{s}^{(\text {medium })}= & -\left(\frac{2 g_{\sigma N N}^{2}}{\pi^{2} m_{\sigma}^{2}}\right) \int_{0}^{\infty}|\vec{p}|^{2} d|\vec{p}|\left[m_{N}^{*}\left(\tilde{C}_{1}^{+p}+\tilde{C}_{1}^{-p}\right)+\frac{2}{3} m_{N}^{*}(e B)^{2}|\vec{p}|^{2}\left(\tilde{C}_{3}^{+p}+\tilde{C}_{3}^{-p}\right)\right. \\
& \left.+2\left(m_{N}^{* 2}+\frac{2}{3}|\vec{p}|^{2}\right)\left\{m_{N}^{*}\left(\kappa_{\mathrm{p}} B\right)^{2}+m_{N}^{*}\left(\kappa_{\mathrm{n}} B\right)^{2}+(|e| B)\left(\kappa_{\mathrm{p}} B\right)\right\}\left(\tilde{C}_{2}^{+p}+\tilde{C}_{2}^{-p}\right)\right] .
\end{aligned}
$$

[1] D. Kharzeev, K. Landsteiner, A. Schmitt, and H.-U. Yee, Lect. Notes Phys. 871, 1 (2013).

[2] J. M. Lattimer and M. Prakash, Phys. Rep. 442, 109 (2007).

[3] E. J. Ferrer, V. de la Incera, and C. Manuel, Phys. Rev. Lett. 95, 152002 (2005); Nucl. Phys. B747, 88 (2006).

[4] E. J. Ferrer and V. de la Incera, Phys. Rev. D 76, 045011 (2007).

[5] K. Fukushima and H. J. Warringa, Phys. Rev. Lett. 100, 032007 (2008).

[6] J. L. Noronha and I. A. Shovkovy, Phys. Rev. D 76, 105030 (2007).

[7] B. Feng, D.-F. Hou, H.-C. Ren, and P.-P. Wu, Phys. Rev. Lett. 105, 042001 (2010).

[8] S. Fayazbakhsh and N. Sadooghi, Phys. Rev. D 82, 045010 (2010); 83, 025026 (2011).

[9] V. Skokov, A. Y. Illarionov, and V. Toneev, Int. J. Mod. Phys. A 24, 5925 (2009).

[10] T. Vachaspati, Phys. Lett. B 265, 258 (1991).

[11] M. D'Elia, S. Mukherjee, and F. Sanfilippo, Phys. Rev. D 82, 051501 (2010).

[12] M. D'Elia and F. Negro, Phys. Rev. D 83, 114028 (2011).

[13] J. K. Boomsma and D. Boer, Phys. Rev. D 81, 074005 (2010).

[14] B. Chatterjee, H. Mishra, and A. Mishra, Phys. Rev. D 84, 014016 (2011).

[15] S. S. Avancini, D. P. Menezes, and C. Providencia, Phys. Rev. C 83, 065805 (2011).

[16] M. Frasca and M. Ruggieri, Phys. Rev. D 83, 094024 (2011).

[17] A. Rabhi and C. Providencia, Phys. Rev. C 83, 055801 (2011).

[18] R. Gattto and M. Ruggieri, Phys. Rev. D 82, 054027 (2010); 83, 034016 (2011).

[19] K. Kashiwa, Phys. Rev. D 83, 117901 (2011).
[20] J. O. Andersen and R. Khan, Phys. Rev. D 85, 065026 (2012).

[21] J. O. Andersen and A. Tranberg, J. High Energy Phys. 08 (2012) 002.

[22] A. J. Mizhar, M. N. Chernodub, and E. S. Fraga, Phys. Rev. D 82, 105016 (2010).

[23] V. Skokov, Phys. Rev. D 85, 034026 (2012).

[24] D. C. Duarte, R. L. S. Farias, and R. O. Ramos, Phys. Rev. D 84, 083525 (2011).

[25] E. S. Fraga and L. F. Palhares, Phys. Rev. D 86, 016008 (2012).

[26] G. S. Bali, F. Bruckmann, G. Endrodi, Z. Fodor, S. D. Katz, S. Krieg, A. Schafer, and K. K. Szabo, J. High Energy Phys. 02 (2012) 044.

[27] G. S. Bali, F. Bruckmann, G. Endrodi, Z. Fodor, S. D. Katz, and A. Schafer, Phys. Rev. D 86, 071502 (2012).

[28] G. S. Bali, F. Bruckmann, G. Endrodi, F. Gruber, and A. Schaefer, J. High Energy Phys. 04 (2013) 130.

[29] V. G. Bornyakov, P. V. Buividovich, N. Cundy, O. A. Kochetkov, and A. Schfer, Phys. Rev. D 90, 034501 (2014).

[30] F. Bruckmann, G. Endrodi, and T. G. Kovacs, J. High Energy Phys. 04 (2013) 112.

[31] J. O. Andersen and W. R. Naylor, Rev. Mod. Phys. 88, 025001 (2016).

[32] S. Mao, Phys. Lett. B 758, 195 (2016).

[33] A. Haber, F. Preis, and A. Schmitt, Phys. Rev. D 90, 125036 (2014).

[34] J. D. Walecka, Ann. Phys. (N.Y.) 83, 491 (1974).

[35] A. Broderick, M. Prakash, and J. M. Lattimer, Astrophys. J. 537, 351 (2000).

[36] A. E. Broderick, M. Prakash, and J. M. Lattimer, Phys. Lett. B 531, 167 (2002).

[37] M. Sinha, B. Mukhopadhyay, and A. Sedrakian, Nucl. Phys. A898, 43 (2013). 
[38] A. Rabhi, P. K. Panda, and C. Providncia, Phys. Rev. C 84, 035803 (2011).

[39] V. Dexheimer, R. Negreiros, and S. Schramm, Eur. Phys. J. A 48, 189 (2012).

[40] F. Preis, A. Rebhan, and A. Schmitt, J. Phys. G 39, 054006 (2012).

[41] J. Dong, W. Zuo, and J. Gu, Phys. Rev. D 87, 103010 (2013).

[42] R. C. R. de Lima, S. S. Avancini, and C. Providncia, Phys. Rev. C 88, 035804 (2013).

[43] R. Casali, L. B. Castro, and D. P. Menezes, Phys. Rev. C 89, 015805 (2014).

[44] F. Preis, A. Rebhan, and A. Schmitt, J. High Energy Phys. 03 (2011) 033.

[45] E. J. Ferrer, V. de la Incera, D. M. Paret, A. P. Martinez, and A. Sanchez, Phys. Rev. D 91, 085041 (2015).

[46] A. Ayla, A. Sanchez, G. Piccinelli, and S. Sahu, Phys. Rev. D 71, 023004 (2005).

[47] J. F. Nieves, Phys. Rev. D 70, 073001 (2004).
[48] J. F. Nieves and P. B. Pal, Phys. Rev. D 73, 105003 (2006).

[49] A. Bandyopadhyay and S. Mallik, Phys. Rev. D 95, 074019 (2017).

[50] S. Mallik and S. Sarkar, Hadrons at Finite Temperature (Cambridge University Press, Cambridge, England, 2016).

[51] J. C. D’Olivo, J. F. Nieves, and S. Sahu, Phys. Rev. D 67, 025018 (2003).

[52] B. D. Serot and J. D. Walecka, in Advances in Nuclear Physics, edited by J. Negele and E. Vogt (Plenum, New York, 1986), p. 1327.

[53] J. Alam, S. Sarkar, P. Roy, T. Hatsuda, and B. Sinha, Ann. Phys. (N.Y.) 286, 159 (2000).

[54] M. Le Bellac, Thermal Field Theory (Cambridge University Press, Cambridge, England, 1996).

[55] R. M. Aguirre and A. L. De Paoli, Eur. Phys. J. A 52, 343 (2016).

[56] R. M. Aguirre, Phys. Rev. D 95, 074029 (2017).

[57] M. E. Peskin and D. V. Schroeder, An Introduction to Quantum Field Theory (Addison-Wesley, Reading, MA, 1995). 